\title{
Flavour symmetries in the SMEFT
}

\author{
Darius A. Faroughy, ${ }^{a}$ Gino Isidori, ${ }^{a}$ Felix Wilsch ${ }^{a}$ and Kei Yamamoto ${ }^{a, b}$ \\ ${ }^{a}$ Physik-Institut, Universität Zürich, \\ CH-8057 Zürich, Switzerland \\ ${ }^{b}$ Graduate School of Science, Hiroshima University, \\ Higashi-Hiroshima 739-8526, Japan \\ E-mail: faroughy@physik.uzh.ch, isidori@physik.uzh.ch, \\ felix.wilsch@physik.uzh.ch, keiy@hiroshima-u.ac.jp
}

AbStRact: We analyse how $\mathrm{U}(3)^{5}$ and $\mathrm{U}(2)^{5}$ flavour symmetries act on the Standard Model Effective Field Theory, providing an organising principle to classify the large number of dimension-six operators involving fermion fields. A detailed counting of such operators, at different order in the breaking terms of both these symmetries, is presented. A brief discussion about possible deviations from these two reference cases, and a simple example of the usefulness of this classification scheme for high- $p_{T}$ analyses at the LHC, are also presented.

KEYwords: Effective Field Theories, Global Symmetries

ARXIV EPRINT: 2005.05366 


\section{Contents}

1 Introduction 1

2 The $\mathrm{U}(3)^{5}$ symmetry and MFV 3

2.1 Minimal Flavour Violation 5

$\begin{array}{lll}2.2 & \text { Summary and discussion } & 7\end{array}$

3 The U(2) ${ }^{5}$ symmetry $\quad 8$

$\begin{array}{llr}3.1 & \text { Fermion bilinears } & 10\end{array}$

$\begin{array}{lll}3.2 & \text { Four fermion operators } & 12\end{array}$

$\begin{array}{lll}3.3 & \text { Summary and discussion } & 16\end{array}$

4 Beyond $\mathrm{U}(3)^{5}$ and $\mathrm{U}(2)^{5}$ with minimal breaking $\quad 17$

$\begin{array}{lll}4.1 & \text { Unbroken } \mathrm{U}(3)_{d, e} \text { groups } & 17\end{array}$

$4.2 \mathrm{U}(2)^{5} \otimes \mathrm{U}(1)_{b} \otimes \mathrm{U}(1)_{\tau} \quad 18$

$\begin{array}{lll}4.3 & \text { Classification of all possible } \mathrm{U}(2)^{5} \text { breaking terms } & 19\end{array}$

5 A phenomenological application: LFV at the LHC 20

6 Conclusions 23

$\begin{array}{ll}\text { A Diagonalization of the Yukawa matrices in } \mathrm{U}(2)^{5} & 25\end{array}$

$\begin{array}{ll}B & \text { On the number of independent fermion contractions }\end{array}$

$\begin{array}{lr}\text { C Summary tables } & 29\end{array}$

\section{Introduction}

Since its conception in the early 70s, the Standard Model (SM) has been regarded as the low-energy limit of an extended theory that includes more degrees of freedom, around or above the electroweak scale, addressing some of its open issues. After the first years of running of the LHC we can state with confidence that there is a mass gap between the SM spectrum and these hypothetical, but still highly motivated, additional degrees of freedom. How large is this mass gap, is probably the most interesting open question nowadays in high-energy physics.

The observation of a mass gap above the SM spectrum, and the need to describe in general terms possible physics beyond the SM, has motivated the systematic study of what goes under the name of SMEFT: the Effective Field Theory (EFT) based on the $\mathrm{SU}(3)_{c} \otimes \mathrm{SU}(2)_{L} \otimes \mathrm{U}(1)_{Y}$ local symmetry, and the SM field content (including the Higgs) 
as dynamical degrees of freedom below a cut-off scale $\Lambda>G_{F}^{-1 / 2}$. Several years after the pioneering analysis in [1], the first complete non-redundant classification of baryonand lepton-number conserving dimension-six operators in the SMEFT has been presented in [2]. Employing such basis, the Renormalization Group (RG) evolution of the Wilson coefficients of these operators, at the one-loop level, has been analysed in [3-5]. ${ }^{1}$

While the number of independent electroweak structures amounts to less than one hundred terms, a large proliferation in the number of independent terms (and corresponding coefficients) in the SMEFT occurs when all the possible flavour structures are taken into account: in absence of any flavour symmetry, they amount to $1350 \mathrm{CP}$-even and $1149 \mathrm{CP}$ odd independent coefficients for the dimension-six operators [5]. The purpose of the present paper is to analyse how a series of motivated hypothesis about flavour symmetries and symmetry-breaking terms can help reduce, and order via an appropriate power counting, such large number of independent terms.

The need of specific hypothesis about symmetry and symmetry-breaking in the flavour sector plays an important role in addressing the key question of how large is the mass gap above the SM spectrum, or the cut-off scale of the SMEFT. In a naïve flavour-anarchic approach, the bounds on the dimension-six operators are dominated by those contributing at tree-level to flavour-violating observables, in particular to $\Delta F=2$ and lepton-flavour violating processes. These operators set bounds of $\mathcal{O}\left(10^{5}\right) \mathrm{TeV}$ on $\Lambda$ for $\mathcal{O}(1)$ coefficients [13]. If this high scale were the overall cut-off scale of the SMEFT, it would imply a severe fine-tuning problem on the Higgs mass term, and it would also imply that most of the operators in the SMEFT play an irrelevant role in current experiments (making the whole construction impractical from the phenomenological point of view). On the other hand, from the known structure of the SM Yukawa couplings, we know that flavour is highly non generic, at least in the dimension-four sector of the EFT. It is therefore natural to employ specific hypothesis about flavour symmetry and symmetry-breaking terms on the whole SMEFT. This procedure has multiple advantages: i) it allows us to lower the overall cutoff scale of the EFT, ameliorating the fine-tuning problem on the Higgs mass; ii) it reduces the number of independent parameters; iii) it makes the EFT construction more consistent and somehow more "appealing", with competing constraints from flavour-conserving and flavour-violating processes on a given effective operator.

The price to pay for this series of advantages is the choice of the flavour symmetry (and symmetry-breaking sector), which necessarily introduces some model dependence. However, if we are interested in symmetries and symmetry-breaking patterns able to successfully reproduce the SM Yukawa couplings and, at the same time, suppress non-standard contributions to flavour-violating observables, the choice is limited. In this paper we focus on two main cases which are particularly motivated from this point of view, i.e. the flavour symmetries $\mathrm{U}(3)^{5}$ and $\mathrm{U}(2)^{5}$, with possible minor variations. The $\mathrm{U}(3)^{5}$ flavour symmetry is the maximal flavour symmetry allowed by the SM gauge group, while $\mathrm{U}(2)^{5}$ is the corresponding subgroup acting only on the first two (light) generations. The $\mathrm{U}(3)^{5}$ symmetry allows us

\footnotetext{
${ }^{1}$ See e.g. [6-12] for recent reviews and phenomenological analyses of the SMEFT with special emphasis on flavour observables.
} 
to implement the Minimal Flavour Violation (MFV) hypotheses $[14,15]$, which is the most restrictive consistent hypothesis we can utilize in the SMEFT to suppress non-standard contributions to flavour-violating observables [15]. The $\mathrm{U}(2)^{5}$ symmetry with minimal breaking [16-18] is quite interesting since it retains most of the MFV virtues, but it allows us to have a much richer structure as far as third-generation dynamics is concerned. For instance, as pointed out in [19-21], the $\mathrm{U}(2)^{5}$ setup provides a very efficient EFT description of the recent flavour anomalies, which cannot be accommodated within a MFV framework.

It must be stressed that the flavour symmetries we are considering are not necessarily fundamental symmetries of the ultraviolet (UV) theory. They could well be accidental symmetries, associated e.g. to some underlying dynamics that act in a non-universal way on the different generations. From the EFT point of view, we cannot distinguish between fundamental or dynamical symmetries in the UV: both of them are effectively described by imposing a specific (global) flavour symmetry in the EFT and specifying a well-defined set of symmetry-breaking terms (the spurions). This effective approach is the one we employ in the present analysis.

It is clear that the $\mathrm{U}(3)^{5}$ and $\mathrm{U}(2)^{5}$ symmetries are not the only options to efficiently suppress flavour-violating observables in the SMEFT. An interesting alternative is provided by the ample class of models based on U(1) symmetries à la Froggatt-Nielsen [22]. ${ }^{2}$ However, on the one hand non-Abelian symmetries are more predictive in establishing a link between SM Yukawa couplings and flavour-violating effects in the SMEFT. On the other hand, in the absence of suprions of non-Abelian groups, a classification of operators such as the one presented here is not particularly illuminating. This is why in this paper we restrict the attention to the case of $\mathrm{U}(3)^{5}$ and $\mathrm{U}(2)^{5}$ symmetries with minimal breaking.

The paper is organised as follows. In section 2 we analyse the $\mathrm{U}(3)^{5}$ symmetry and the MFV hypothesis. In section 3 we analyse the $\mathrm{U}(2)^{5}$ symmetry with minimal breaking. A general discussion about possible deviations from these two reference cases, with the specific analysis of the impact of $\mathrm{U}(1)$ symmetries acting on third-generation down-quarks and/or charged leptons, is presented in section 4 . In section 5 we briefly illustrate the usefulness of this approach in high- $p_{T}$ phenomenological analyses at the LHC, choosing $p p \rightarrow \ell \tau$ $(\ell=e, \mu)$ as a representative example. The results are summarised in the Conclusions.

\section{The $\mathrm{U}(3)^{5}$ symmetry and MFV}

The largest group of flavour-symmetry transformations compatible with the gauge symmetries of the SM Lagrangian is [14, 24]

$$
G_{\text {flavour }}=\mathrm{U}(3)^{5}=\mathrm{U}(3)_{\ell} \otimes \mathrm{U}(3)_{q} \otimes \mathrm{U}(3)_{e} \otimes \mathrm{U}(3)_{u} \otimes \mathrm{U}(3)_{d}=\mathrm{SU}(3)^{5} \otimes \mathrm{U}(1)^{5},
$$

where, with a standard notation, $\{\ell, q, e, u, d\}$ denote the five independent types of SM fermions with different gauge quantum numbers:

$$
\mathcal{L}_{\mathrm{SM}}^{\mathrm{fermions}}=\sum_{\psi=\ell, q, e, u, d} \bar{\psi} i \not D \psi+\left(\bar{\ell} Y_{e} e H+\bar{q} Y_{d} d H+\bar{q} Y_{u} u H_{c}+\text { h.c. }\right) .
$$

\footnotetext{
${ }^{2}$ An interesting systematic analysis about the implementation of U(1) symmetries in the SMEFT, together with general dynamical assumptions about new physics, has recently been presented in ref. [23].
} 
Within the SM, the Yukawa couplings $\left(Y_{e, u, d}\right)$ are the only source of breaking of $G_{\text {flavour }}$. They break this global symmetry as follows

$$
G_{\text {flavour }}=\left\{\begin{array}{lll}
\mathrm{SU}(3)^{5} & Y_{e, u, d} \neq 0 \\
\mathrm{U}(1)^{5} & \mathrm{U}(1)_{e-\mu} \otimes \mathrm{U}(1)_{\tau-\mu} \\
& \mathrm{U}(1)_{B} \otimes \mathrm{U}(1)_{L} \otimes \mathrm{U}(1)_{Y}
\end{array}\right.
$$

where we separated explicitly flavour-universal and flavour-non-universal subgroups. The three unbroken flavour-universal U(1) groups are baryon number, lepton number, and hypercharge. ${ }^{3}$

As anticipated, our goal is to count and classify the number of independent dimensionsix operators, and corresponding effective couplings, in the SMEFT according to different hypotheses about the breaking of $G_{\text {flavour }}$. The most restrictive assumption we can make is that $G_{\text {flavour }}$ is an exact symmetry of the beyond-the-SM sector. This assumption is not fully consistent, since $G_{\text {flavour }}$ is broken within the SM. However, it is a useful starting point for the classification of the operators, and it is a coherent hypothesis to be implemented in the SMEFT in the limit where we neglect $G_{\text {flavour }}$ breaking terms also in the SM sector, i.e. in the limit where we neglect the SM Yukawa couplings.

In this section we compare the results obtained in this limit (i.e. the exact $\mathrm{U}(3)^{5}$ limit), as well as those obtained under the MFV hypothesis (considering the first few terms in the expansion in powers of Yukawa couplings), to those obtained in the absence of any flavour symmetry assuming one or three generations of SM fermions.

Exact $\mathbf{U}(3)^{\mathbf{5}}$ symmetry. To classify the SMEFT operators we adopt the Warsaw basis [2], whose notation to identify the different electroweak structures, adopting the division in classes introduced in [5], will be followed throughout the whole paper. ${ }^{4}$ The operators of classes 1-4 do not contain fermions, thus the counting of independent couplings is trivial: 9 independent CP-even coefficients for the 9 hermitian structures and $6 \mathrm{CP}$-odd coefficients for the anti-hermitian ones. The operators of the classes 5 and 6 are forbidden in the exact $\mathrm{U}(3)^{5}$ limit, since they contain a fermion current of the type $\bar{L} R$. The class 7 operators, but for $Q_{H u d}$, are all hermitian and allowed, provided the fermion indices are properly summed, hence they contribute one real coefficient each. The operator $Q_{H u d}$ is forbidden.

For the operators containing four fermions we have three structures which are hermitian, namely $(\bar{L} L)(\bar{L} L),(\bar{R} R)(\bar{R} R)$ and $(\bar{L} L)(\bar{R} R)$, which are allowed by the symmetry provided the fermion indices are properly summed. Here each operator corresponds to one real coefficient, with the exception of $Q_{\ell \ell}, Q_{q q}^{(1)}, Q_{q q}^{(3)}, Q_{u u}$ and $Q_{d d}$, which corresponds to two independent operators (hence two real coefficients) since there are two independent $\mathrm{U}(3)^{5}$-invariant ways to contract the flavour indices. For example, in the case of $Q_{\ell \ell}$ we find

$$
\left(\bar{\ell}_{p} \gamma_{\mu} \ell_{p}\right)\left(\bar{\ell}_{r} \gamma_{\mu} \ell_{r}\right) \quad \text { and } \quad\left(\bar{\ell}_{p} \gamma_{\mu} \ell_{r}\right)\left(\bar{\ell}_{r} \gamma_{\mu} \ell_{p}\right)
$$

\footnotetext{
${ }^{3}$ The two flavour-non-universal U(1) subgroups left unbroken by the Yukawa couplings are arbitrary combinations of the two diagonal generators of $\mathrm{SU}(3)_{e+\ell}$, namely the vectorial subgroup of $\mathrm{SU}(3)_{e} \otimes \mathrm{SU}(3)_{\ell}$.

${ }^{4}$ To facilitate the readability of this paper, the complete list of operators containing fermion fields is reported in table 8 of appendix $\mathrm{C}$.
} 


\begin{tabular}{|cl|cc|ccc|cc|cc|c|}
\hline \multirow{2}{*}{ Class } & Operators & \multicolumn{4}{|c|}{ No symmetry } & \multicolumn{5}{c|}{$\mathrm{U}(3)^{5}$} \\
\hline $1-4$ & $X^{3}, H^{6}, H^{4} D^{2}, X^{2} H^{2}$ & 9 & 6 & 9 & 6 & 9 & 6 & 9 & 6 & 9 & 6 \\
\hline 5 & $\psi^{2} H^{3}$ & 27 & 27 & 3 & 3 & - & - & 3 & 3 & 4 & 4 \\
\hline 6 & $\psi^{2} X H$ & 72 & 72 & 8 & 8 & - & - & 8 & 8 & 11 & 11 \\
7 & $\psi^{2} H^{2} D$ & 51 & 30 & 8 & 1 & 7 & - & 7 & - & 11 & 1 \\
\hline \multirow{2}{*}{$(\bar{L} L)(\bar{L} L)$} & 171 & 126 & 5 & - & 8 & - & 8 & - & 14 & - \\
& $(\bar{R} R)(\bar{R} R)$ & 255 & 195 & 7 & - & 9 & - & 9 & - & 14 & - \\
8 & $(\bar{L} L)(\bar{R} R)$ & 360 & 288 & 8 & - & 8 & - & 8 & - & 18 & - \\
& $(\bar{L} R)(\bar{R} L)$ & 81 & 81 & 1 & 1 & - & - & - & - & - & - \\
& $(\bar{L} R)(\bar{L} R)$ & 324 & 324 & 4 & 4 & - & - & - & - & 4 & 4 \\
\hline \multirow{2}{*}{ total: } & 1350 & 1149 & 53 & 23 & 41 & 6 & 52 & 17 & 85 & 26 \\
\hline
\end{tabular}

Table 1. Number of independent operators in $\mathrm{U}(3)^{5}$, MFV and without symmetry. In each column the left (right) number corresponds to the number of CP-even (CP-odd) coefficients. $\mathcal{O}\left(X^{n}\right)$ stands for including terms up to $\mathcal{O}\left(X^{n}\right)$.

where $r$ and $p$ denote the flavour indices (and the sum over repeated indices is understood). Note that the $Q_{e e}$ operator only corresponds to a single independent structure due to the Fierz identity

$$
\left(\bar{e}_{p} \gamma_{\mu} e_{r}\right)\left(\bar{e}_{s} \gamma_{\mu} e_{t}\right)=\left(\bar{e}_{s} \gamma_{\mu} e_{r}\right)\left(\bar{e}_{p} \gamma_{\mu} e_{t}\right)
$$

The operators of the type $(\bar{L} R)(\bar{R} L)$ and $(\bar{L} R)(\bar{L} R)$ are not allowed by the symmetry.

The results thus obtained are reported in table 1 in the "Exact" $\mathrm{U}(3)^{5}$ column: the left (right) value in each entry indicates the number of CP-even (CP-odd) coefficients. For comparison, we also show the counting of independent coefficients if no symmetry is imposed, or if a single generation of fermions is considered, where we fully agree with the results derived first in ref. [5]. The counting in the latter case proceeds in close analogy to the $\mathrm{U}(3)^{5}$ case with a few important differences: the operators in classes 5 and 6 , as well as $Q_{H u d}$ in class 7, which are not hermitian, leads to one real and one imaginary coefficients each. The operators in the $(\bar{L} L)(\bar{L} L)$ and $(\bar{R} R)(\bar{R} R)$ categories which had two possible flavour contractions with more generations, now have only a single contraction, hence in these categories we have one real coefficient for each electroweak structure. Finally, for the non-hermitian operators of the type $(\bar{L} R)(\bar{R} L)$ and $(\bar{L} R)(\bar{L} R)$ we can identify one real and one imaginary coefficient for each electroweak structure.

\subsection{Minimal Flavour Violation}

The MFV hypothesis is the assumption that the SM Yukawa couplings are the only sources of $\mathrm{U}(3)^{5}$ breaking $[14,15]$. The exact $\mathrm{U}(3)^{5}$ limit analysed before is equivalent to employing the MFV hypothesis and working to zeroth order in the symmetry breaking terms. To go beyond leading order we promote the SM Yukawa couplings to $\mathrm{U}(3)^{5}$ spurion fields with 
the following transformation properties [15]:

$$
Y_{u}=(1,3,1, \overline{3}, 1), \quad Y_{d}=(1,3,1,1, \overline{3}), \quad Y_{e}=(3,1, \overline{3}, 1,1) .
$$

In principle, the spurions can appear with arbitrary powers both in the renormalizable $(d=4)$ part of the Lagrangian and in the dimension-six effective operators. However, via a suitable redefinition of both fermion fields and spurions, we can always put the $d=4$ Lagrangian to its standard expression in eq. (2.2), namely we can always identify the spurions with the SM Yukawa couplings. This implies we can always choose a flavour basis where the spurions are completely determined in terms of fermion masses and the CabibboKobayashi-Maskawa (CKM) matrix, $V_{\text {CKM }}$. A representative example is the down-quark mass-eigenstate basis, where

$$
Y_{e}=\operatorname{diag}\left(y_{e}, y_{\mu}, y_{\tau}\right), \quad Y_{d}=\operatorname{diag}\left(y_{d}, y_{s}, y_{b}\right), \quad Y_{u}=V_{\mathrm{CKM}}^{\dagger} \times \operatorname{diag}\left(y_{u}, y_{c}, y_{t}\right) .
$$

The key point is that there are no free (observable) parameters in the structure of the MFV spurions. As we shall see, this is not the case for less restrictive symmetry hypotheses, such as the $\mathrm{U}(2)^{5}$ case discussed in section 3 . We are now ready to count the number of independent operators appearing at $d=6$ in the SMEFT inserting a small number of symmetry breaking terms.

Terms of $\mathcal{O}\left(Y_{u, d, e}\right)$. With a single insertions of the Yukawa couplings, only the operators in class 5 and 6 gets modified with respect to the $\mathrm{U}(3)^{3}$ invariant case: as far as the flavour structure is concerned, these operators are identical to the three Yukawa interactions in eq. (2.2). Since they are not hermitian, we get 3 (8) CP-even and 3 (8) CP-odd parameters for $\psi^{2} H^{3}+$ h.c. $\left(\psi^{2} X H+\right.$ h.c.). The counting of independent terms thus obtained, reported in table 1 , is consistent with that performed in [25].

Terms of $\mathcal{O}\left(Y_{u}^{2}\right)$. Here the operators involved are those in class 7 and 8 , which contain at least two $q$ fields or two $u$ fields, and that we can conveniently re-arrange in the following three categories

A) Operators with a bilinear current of the type $\bar{q} \Gamma q$ or $\bar{u} \Gamma u$ :

3 in class 7: $Q_{H q}^{(1,3)}$ and $Q_{H u}$

2 in class $(\bar{L} L)(\bar{L} L): Q_{\ell q}^{(1,3)}$

3 in class $(\bar{R} R)(\bar{R} R): Q_{e u}$ and $Q_{u d}^{(1,8)}$

4 in class $(\bar{L} L)(\bar{R} R): Q_{\ell u}, Q_{q e}, Q_{q d}^{(1,8)}$

B) Operators of the type $\bar{q} \Gamma q \times \bar{u} \Gamma u$ :

2 in class $(\bar{L} L)(\bar{R} R): Q_{q u}^{(1,8)}$

C) Operators with four $u$ or four $q$ fields:

2 in class $(\bar{L} L)(\bar{L} L): Q_{q q}^{(1,3)}$

1 in class $(\bar{R} R)(\bar{R} R): Q_{u u}$ 
where $\Gamma$ denote a generic combination of Dirac matrices, color and $\mathrm{SU}(2)_{L}$ generators, which play no role as far as the flavour structure is concerned. For the operators in the category A) we obtain a $\mathrm{U}(3)^{5}$ singlet contracting $Y_{u}$ and $Y_{u}^{\dagger}$ to form an octet of $\mathrm{SU}(3)_{u}$ or $\mathrm{SU}(3)_{q}$, and then contracting this octet with the flavour indices of the $q$ - or $u$-quark current (the other current being necessarily a flavour singlet):

$$
\bar{q}_{p} \Gamma q_{r}\left(Y_{u} Y_{u}^{\dagger}\right)_{p r}, \quad \bar{u}_{p} \Gamma u_{r}\left(Y_{u}^{\dagger} Y_{u}\right)_{p r} .
$$

Thus all the hermitian structures in the category A) yield one CP-even coupling. For the operators in the category B) three contractions are possible:

$$
\left(\bar{u}_{a} \Gamma u_{a}\right)\left(\bar{q}_{p} \Gamma q_{r}\right)\left(Y_{u} Y_{u}^{\dagger}\right)_{p r}, \quad\left(\bar{u}_{p} \Gamma u_{r}\right)\left(\bar{q}_{a} \Gamma q_{a}\right)\left(Y_{u}^{\dagger} Y_{u}\right)_{p r}, \quad\left(\bar{u}_{p} \Gamma u_{s}\right)\left(\bar{q}_{r} \Gamma q_{t}\right)\left(Y_{u}^{\dagger}\right)_{p t}\left(Y_{u}\right)_{r s},
$$

Thus all the hermitian structures in the category B) yield three CP-even couplings. Finally, for the operators in the category $\mathrm{C}$ ) only two contractions are possible, such as

$$
\left(\bar{q}_{a} \Gamma q_{a}\right)\left(\bar{q}_{p} \Gamma q_{r}\right)\left(Y_{u} Y_{u}^{\dagger}\right)_{p r}, \quad\left(\bar{q}_{p} \Gamma q_{a}\right)\left(\bar{q}_{a} \Gamma q_{r}\right)\left(Y_{u} Y_{u}^{\dagger}\right)_{p r},
$$

and similarly for the $u$ fields. All other contractions either reduce to those or to genuine singlet contractions that have already been counted in the $\mathrm{U}(3)^{5}$ invariant case. Thus all the hermitian structures in the category C) yield two CP-even couplings. Summing up, we find the following CP-even couplings for operators with two powers of $Y_{u}$ : 3 in class 7, 6 in class $(\bar{L} L)(\bar{L} L), 5$ in class $(\bar{R} R)(\bar{R} R)$ and 10 in class $(\bar{L} L)(\bar{R} R)$.

Terms of $\mathcal{O}\left(\boldsymbol{Y}_{\boldsymbol{u}} \boldsymbol{Y}_{\boldsymbol{d}}\right)$ and $\mathcal{O}\left(\boldsymbol{Y}_{u}^{\mathbf{2}} \boldsymbol{Y}_{\boldsymbol{d}}\right)$. Proceeding in a similar manner we can identify the independent terms with one $Y_{d}$ and one or two $Y_{u}$ spurions. Three non-hermitian structures can have flavour-singlet contractions with one $Y_{d}$ and one $Y_{u}: Q_{H u d}\left(1\right.$ possibility), $Q_{q u q d}^{(1)}$ and $Q_{q u q d}^{(8)}$ (two possibilities each), for a total of five CP-even and five CP-odd parameters. Inserting two up-type and one down-type spurions, we can form four (non-hermitian) flavour-singlet operators using the structures with one $d$ and one $q$ fields in class 5 and 6 , for a total of four CP-even and four CP-odd parameters.

\subsection{Summary and discussion}

The overall number of independent terms allowed by the MFV hypothesis with at most one "small" Yukawa coupling, namely $Y_{d}$ and $Y_{e}$, and up to two powers of $Y_{u}$ is shown in the last column of table $1 .^{5}$ At this order we have all the operators necessary to describe deviations from the SM in rare flavour-violating processes that do occur within the SM and, within the SM, receive sizeable short-distance contributions induced by the large top-quark mass (such as $B^{0}-\bar{B}^{0}$ and $K^{0}-\bar{K}^{0}$ mixing, $b \rightarrow s \gamma, b \rightarrow s \ell^{+} \ell^{-}, \ldots$ ) [15]. As can be seen, the number of operators at this order is much smaller than that obtained in absence of any symmetry (for three generations) and still remarkably close to the single generation case.

Beside being a very strong hypothesis about the UV completion of the SM, a drawback of the MFV hypothesis is that it does not allow us to define a clear power-counting in the

\footnotetext{
${ }^{5}$ A detailed counting order by order in the insertions of different powers of the Yukawa couplings in presented in table 9 in appendix C.
} 
SMEFT. This is because one of the breaking term, namely $y_{t}$, or better the 33 entries of $Y_{u} Y_{u}^{\dagger}$ and $Y_{u}^{\dagger} Y_{u}$ in the basis (2.7), is large. It is therefore not obvious why one should not consider more powers of $Y_{u}$ in the counting of independent operators, as for instance done in the non-linear realizations proposed in $[26,27]$. However, it is only $y_{t}$ that is large, not the other entries of $Y_{u}$. The insertion of an arbitrary powers of $y_{t}$ triggers the following breaking pattern

$$
\mathrm{U}(3)_{q} \otimes \mathrm{U}(3)_{u} \stackrel{y_{t}}{\longrightarrow} \mathrm{U}(2)_{q} \otimes \mathrm{U}(2)_{u} \otimes \mathrm{U}(1)_{q_{L}^{3}+t_{R}} .
$$

A similar breaking to $\mathrm{U}(2)$ subgroups occurs if we allow the third generation Yukawa couplings of down quarks and charged leptons to be large (a possibility that naturally occurs in models with an extended Higgs sector). This observation, together with the more general argument that the third generation of fermions might play a special role in extensions of the SM, naturally brings us to consider a smaller symmetry group acting only on the light fermion families, that is what we discuss next.

\section{The $\mathrm{U}(2)^{5}$ symmetry}

The $\mathrm{U}(2)^{5}$ symmetry is the subgroup of $\mathrm{U}(3)^{5}$ that, by construction, distinguish the first two generations of fermions from the third one [16-18]. It provides a "natural" explanation of why third-generation Yukawa couplings are large (being allowed by the symmetry) and, contrary to the MFV case, it allows us to build an EFT where all the breaking terms are small, offering a more precise power counting for the operators.

Given a fermion species $\psi_{f}(f=\ell, q, e, u, d)$, the first two generations form a doublet of one of the $\mathrm{U}(2)$ subgroups, whereas $\psi_{f}^{3}$ transform as a singlet. The five independent flavour doublets are denoted $L, Q, E, U, D$ and the flavour symmetry is decomposed as

$$
\mathrm{U}(2)^{5}=\mathrm{U}(2)_{L} \otimes \mathrm{U}(2)_{Q} \otimes \mathrm{U}(2)_{E} \otimes \mathrm{U}(2)_{U} \otimes \mathrm{U}(2)_{D}
$$

A set of symmetry breaking terms able to reproduce the observed SM Yukawa couplings, which is minimal both in terms of the number of independent spurions, as well as in their size, is given by [16]

$$
\begin{aligned}
V_{\ell} & \sim(2,1,1,1,1), & V_{q} & \sim(1,2,1,1,1), \\
\Delta_{e} & \sim(2,1, \overline{2}, 1,1), & \Delta_{u} & \sim(1,2,1, \overline{2}, 1),
\end{aligned}
$$

By construction, $V_{q, \ell}$ are complex two-vectors and $\Delta_{e, u, d}$ are complex $2 \times 2$ matrices. In terms of these spurions, we can express the Yukawa matrices as

$$
Y_{e}=y_{\tau}\left(\begin{array}{cc}
\Delta_{e} & x_{\tau} V_{\ell} \\
0 & 1
\end{array}\right), \quad Y_{u}=y_{t}\left(\begin{array}{cc}
\Delta_{u} & x_{t} V_{q} \\
0 & 1
\end{array}\right), \quad Y_{d}=y_{b}\left(\begin{array}{cc}
\Delta_{d} & x_{b} V_{q} \\
0 & 1
\end{array}\right)
$$

where $y_{\tau, t, b}$ and $x_{\tau, t, b}$ are free complex parameters expected to be of order $\mathcal{O}(1)$. Alternative breaking terms, and the embedding of $\mathrm{U}(2)^{5}$ in $\mathrm{U}(3)^{5}$, are discussed in section 4 . 
Explicit form for the spurions. As already pointed out in the MFV case, the spurions can appear with arbitrary powers both in the renormalizable $(d=4)$ part of the Lagrangian and in the dimension-six effective operators. In this case, we redefine the fields such that the kinetic terms are canonically normalised and the Yukawa couplings assume the form in eq. (3.3). This condition unambiguously normalises the $\Delta$ spurions, but it leaves an $\mathcal{O}(1)$ freedom in the normalisation of the $V$ spurions (encoded by $x_{\tau, t, b}$ ).

Using the residual $\mathrm{U}(2)^{5}$ invariance, we can transform the spurions to the following explicit form

$$
V_{q(\ell)}=e^{i \bar{\phi}_{q(\ell)}}\left(\begin{array}{c}
0 \\
\epsilon_{q(\ell)}
\end{array}\right), \quad \Delta_{e}=O_{e}^{\top}\left(\begin{array}{cc}
\delta_{e}^{\prime} & 0 \\
0 & \delta_{e}
\end{array}\right), \quad \Delta_{u}=U_{u}^{\dagger}\left(\begin{array}{cc}
\delta_{u}^{\prime} & 0 \\
0 & \delta_{u}
\end{array}\right), \quad \Delta_{d}=U_{d}^{\dagger}\left(\begin{array}{cc}
\delta_{d}^{\prime} & 0 \\
0 & \delta_{d}
\end{array}\right)
$$

The flavour basis where the spurions assume this form is what we define as interaction basis for the fermion fields in the $\mathrm{U}(2)^{5}$ setup. Here $O$ and $U$ represent $2 \times 2$ orthogonal and complex unitary matrices, respectively

$$
O_{e}=\left(\begin{array}{cc}
c_{e} & s_{e} \\
-s_{e} & c_{e}
\end{array}\right), \quad U_{q}=\left(\begin{array}{cc}
c_{q} & s_{q} e^{i \alpha_{q}} \\
-s_{q} e^{-i \alpha_{q}} & c_{q}
\end{array}\right)
$$

with $s_{i} \equiv \sin \theta_{i}$ and $c_{i} \equiv \cos \theta_{i}$. The $\epsilon_{i}$ and $\delta_{i}^{(\prime)}$ are small positive real parameters controlling the overall size of the spurions. From the observed hierarchies of the Yukawa couplings, we deduce

$$
1 \gg \epsilon_{i} \gg \delta_{i} \gg \delta_{i}^{\prime}>0
$$

or, more precisely,

$$
\begin{aligned}
\epsilon_{i} & =\mathcal{O}\left(\operatorname{Tr}\left(Y_{u} Y_{u}^{\dagger}\right)-\frac{\operatorname{Tr}\left(Y_{u} Y_{u}^{\dagger} Y_{d} Y_{d}^{\dagger}\right)}{\operatorname{Tr}\left(Y_{d} Y_{d}^{\dagger}\right)}\right)^{1 / 2}=\mathcal{O}\left(y_{t}\left|V_{t s}\right|\right)=\mathcal{O}\left(10^{-1}\right), \\
\delta_{i} & =\mathcal{O}\left(\frac{y_{c}}{y_{t}}, \frac{y_{s}}{y_{b}}, \frac{y_{\mu}}{y_{\tau}}\right)=\mathcal{O}\left(10^{-2}\right) \\
\delta_{i}^{\prime} & =\mathcal{O}\left(\frac{y_{u}}{y_{t}}, \frac{y_{d}}{y_{b}}, \frac{y_{e}}{y_{\tau}}\right)=\mathcal{O}\left(10^{-3}\right) .
\end{aligned}
$$

Starting from the interaction basis, the Yukawa couplings in (3.3) are diagonalized by unitary transformations of the type $L_{f}^{\dagger} Y_{f} R_{f}=\operatorname{diag}\left(Y_{f}\right)$, with $f=u, d$,e. The explicit form of these matrices is reported in appendix A. While the $\delta_{i}^{(\prime)}$ are in one-to-one correspondence with the light Yukawa eigenvalues, not all the other parameters appearing in the Yukawa and spurion decompositions in eqs. (3.3)-(3.5) can be put in correspondence with SM parameters (in particular with CKM elements). Contrary to the MFV case, in the $\mathrm{U}(2)^{5}$ setup the structure of the spurions is not completely determined in terms of known parameters. However, once we impose the hierarchy among the size of the spurions in eq. (3.9), we effectively "protect" quark mixing as in the MFV case [16]. 


\subsection{Fermion bilinears}

We can now proceed classifying the number of independent operators appearing at $d=6$ in the SMEFT with a $\mathrm{U}(2)^{5}$ flavour symmetry, minimally broken as discussed above. Our final goal is to classify the operators up to $\mathcal{O}\left(V^{3}, \Delta^{1} V^{1}\right)$, namely with up to three $V$ spurions (but no $\Delta$ terms), or with one $\Delta$ and at most one $V$. Given the size of the spurions in eq. (3.9), this corresponds to neglecting terms which are at most of $\mathcal{O}\left(10^{-4}\right)$ according to our main hypotheses.

We start the analysis from the operators of classes 5, 6 and 7, which contains a fermion bilinear. To better illustrate how the hypothesis of a minimally broken $\mathrm{U}(2)^{5}$ symmetry acts on the different flavour structures, in the case of left-handed and right-handed bilinears we analyse also the effect of subleading breaking terms up to $\mathcal{O}\left(\Delta^{2} V^{2}\right)$. More precisely, in the following we analyse how to span the flavour structure of the independent fermion bilinears in terms of the $\mathrm{U}(2)^{5}$ breaking spurions.

Left-handed bilinears. As a representative example of left-handed fermion bilinears we discuss in detail the leptonic case (the translation to the quark case being trivial). For simplicity we omit $\mathrm{SU}(2)_{L}$ and spinor indices, and often also flavour indices (except in expressions which would be ambiguous otherwise). The possible terms at different orders in the spurions for the case at hand is shown in table 2 . The results can be summarised as follows in terms of the flavour tensor $\Lambda_{L L}$ :

$$
\bar{\ell}_{p} \Gamma \Lambda_{L L}^{p r} \ell_{r}, \quad \Lambda_{L L}=\left(\begin{array}{ccc}
a_{1} & 0 & 0 \\
0 & a_{1}+c_{1} \epsilon_{\ell}^{2} & \beta_{1} \epsilon_{\ell} \\
0 & \beta_{1}^{*} \epsilon_{\ell} & a_{2}
\end{array}\right)+\mathcal{O}\left(\delta_{e}^{2}\right) .
$$

The explicit expression of $\Lambda_{L L}$ in eq. (3.10) corresponds to the expansion truncated at $\mathcal{O}(\Delta V)$ in the interaction basis. As can be seen, at this order there is no mixing between the first generation and the others: ${ }^{6} \Lambda_{L L}$, that in absence of any flavour symmetry is parameterised by 6 real and 3 imaginary coefficients has only 4 real $\left(a_{1,2}, c_{1}, \operatorname{Re} \beta_{1}\right)$ and 1 imaginary $\left(\operatorname{Im} \beta_{1}\right)$ coefficients. A complete span of the whole $3 \times 3$ hermitian structure of $\Lambda_{L L}$ occurs only with the inclusion of the terms up to $\mathcal{O}\left(\Delta^{2} V^{2}\right)$ shown in the lower part of table 2 .

Here and in the following, when presenting explicit expressions, the phases of nonhermitian spurion combinations are reabsorbed into that of the corresponding complex coefficients. The criteria used to label the different terms are as follows: we denote with latin (greek) letters the real (complex) couplings appearing in hermitian (non-hermitian) structures. Terms with the same number of spurions are denoted with the same latin or greek letter and different subscript. Note that this notation focuses only on the flavour indices and not on the electroweak structure. A complete notation for the coupling of each operator can be chosen of the type $C_{X}(F)$, where $X$ denotes a specific electroweak structure, as in table $8(X=H \ell, H q, \ldots)$, and $F=a_{i}, \beta_{i}, \ldots$ denotes the flavour structure.

\footnotetext{
${ }^{6}$ Note that this statement holds only in the interaction basis.
} 


\begin{tabular}{|l|l|l|}
\hline Spurions & Operator & Explicit expression in flavour components \\
\hline$V^{0}$ & $a_{1} \bar{L} L+a_{2} \bar{\ell}_{3} \ell_{3}$ & $a_{1}\left(\bar{\ell}_{1} \ell_{1}+\bar{\ell}_{2} \ell_{2}\right)+a_{2}\left(\bar{\ell}_{3} \ell_{3}\right)$ \\
$V^{1}$ & $\beta_{1} \bar{L} V_{\ell} \ell_{3}+$ h.c. & $\beta_{1} \epsilon_{\ell}\left(\bar{\ell}_{2} \ell_{3}\right)+$ h.c. \\
$V^{2}$ & $c_{1} \bar{L} V_{\ell} V_{\ell}^{\dagger} L$ & $c_{1} \epsilon_{\ell}^{2}\left(\bar{\ell}_{2} \ell_{2}\right)$ \\
$\Delta^{1}, \Delta^{1} V^{1}$ & - & - \\
\hline$\Delta^{2}$ & $h_{1} \bar{L} \Delta_{e} \Delta_{e}^{\dagger} L$ & $\approx h_{1}\left[\delta_{e}^{2}\left(\bar{\ell}_{2} \ell_{2}\right)-s_{e} \delta_{e}^{2}\left(\bar{\ell}_{1} \ell_{2}+\bar{\ell}_{2} \ell_{1}\right)+\left(s_{e}^{2} \delta_{e}^{2}+\delta_{e}^{\prime 2}\right)\left(\bar{\ell}_{1} \ell_{1}\right)\right]$ \\
$\Delta^{2} V^{1}$ & $\lambda_{1} \bar{L} \Delta_{e} \Delta_{e}^{\dagger} V_{\ell} \ell_{3}+$ h.c. & $\approx \lambda_{1} \epsilon_{\ell} \delta_{e}^{2}\left(\bar{\ell}_{2} \ell_{3}-s_{e} \bar{\ell}_{1} \ell_{3}\right)+$ h.c. \\
$\Delta^{2} V^{2}$ & $\mu_{1} \bar{L} \Delta_{e} \Delta_{e}^{\dagger} V_{\ell} V_{\ell}^{\dagger} L+$ h.c. & $\approx \mu_{1} \epsilon_{\ell}^{2} \delta_{e}^{2}\left(\bar{\ell}_{2} \ell_{2}-s_{e} \bar{\ell}_{1} \ell_{2}\right)+$ h.c. \\
\hline
\end{tabular}

Table 2. Left-handed fermion bilinears allowed by different $U(2)$ breaking terms. The terms below the horizontal line are subleading structures which are not considered in the general analysis of independent terms. The expressions in the third column are expanded in powers of $s_{e}$ up to first non-vanishing terms.

Right-handed bilinears. Proceeding in a similar manner, in table 3 we report righthanded fermion bilinears which are allowed by different spurion combinations. The leptonic bilinear $\bar{e} e$ is representative of any right-handed fermion bilinear with identical fields, while we treated separately the $\bar{u} d$ case which appears only for the operator $Q_{H u d}$. As far as identical fermions are concerned, we can express the result via the flavour tensor $\Lambda_{R R}$ :

$$
\bar{e}_{p} \Gamma \Lambda_{R R}^{p r} e_{r}, \quad \Lambda_{R R}=\left(\begin{array}{ccc}
a_{1} & 0 & \sigma_{1}^{*} \epsilon_{\ell} s_{e} \delta_{e}^{\prime} \\
0 & a_{1} & \sigma_{1}^{*} \epsilon_{\ell} \delta_{e} \\
\sigma_{1} \epsilon_{\ell} s_{e} \delta_{e}^{\prime} & \sigma_{1} \epsilon_{\ell} \delta_{e} & a_{2}
\end{array}\right)+\mathcal{O}\left(\delta_{e}^{2}\right)
$$

Terminating the expansion up to $\mathcal{O}(\Delta V), \Lambda_{R R}$ contains 3 real and 1 imaginary coefficients. At the same order, in the case of the (non-hermitian) $\bar{u} d$ bilinear one finds 3 real and 3 imaginary coefficients (see table 3 ).

Interestingly, this structure is quite "robust" with respect to higher-order corrections. At $\mathcal{O}\left(\Delta^{2}\right)$ one generates a difference between the 11 and 22 entires of $\Lambda_{R R}$, and only at $\mathcal{O}\left(\Delta^{2} V^{2}\right)$ non-vanishing 12 and 21 entries, but this is not enough to span the entire $3 \times 3$ hermitian structure: this goal can be achieved only with inclusion of $\mathcal{O}\left(\Delta^{4} V^{2}\right)$ terms. Most important, mixing terms involving first and/or second generations always require a suppression factor proportional to $\delta_{f}$ and/or $\delta_{f}^{\prime}$. This is a feature related to our minimal choice of breaking terms.

Left-right bilinears. The independent flavour structures of left-right fermion bilinear are listed in table 4, where we focus on the leptonic sector as representative example. 


\begin{tabular}{|l|l|l|}
\hline Spurions & Operator $(\bar{e} e$ type $)$ & Explicit expression in flavour components \\
\hline$V^{0}$ & $a_{1} \bar{E} E+a_{2} \bar{e}_{3} e_{3}$ & $a_{1}\left(\bar{e}_{1} e_{1}+\bar{e}_{2} e_{2}\right)+a_{2}\left(\bar{e}_{3} e_{3}\right)$ \\
$V^{1}, V^{2}, \Delta^{1}$ & - & \\
$\Delta^{1} V^{1}$ & $\sigma_{1} \bar{e}_{3} V_{\ell}^{\dagger} \Delta_{e} E+$ h.c. & $\approx \sigma_{1} \epsilon_{\ell}\left[\delta_{e}\left(\bar{e}_{3} e_{2}\right)+s_{e} \delta_{e}^{\prime}\left(\bar{e}_{3} e_{1}\right)\right]+$ h.c. \\
\hline$\Delta^{2}$ & $h_{1} \bar{E} \Delta_{e}^{\dagger} \Delta_{e} E$ & $h_{1}\left[\delta_{e}^{2}\left(\bar{e}_{2} e_{2}\right)+\delta_{e}^{\prime 2}\left(\bar{e}_{1} e_{1}\right)\right]$ \\
$\Delta^{2} V^{1}$ & - & \\
$\Delta^{2} V^{2}$ & $m_{1} \bar{E} \Delta_{e}^{\dagger} V_{\ell} V_{\ell}^{\dagger} \Delta_{e} E$ & $\approx m_{1} \epsilon_{\ell}^{2}\left[\delta_{e}^{2}\left(\bar{e}_{2} e_{2}\right)+s_{e} \delta_{e}^{\prime} \delta_{e}\left(\bar{e}_{1} e_{2}+\bar{e}_{2} e_{1}\right)+s_{e}^{2} \delta_{e}^{\prime 2}\left(\bar{e}_{1} e_{1}\right)\right]$ \\
\hline
\end{tabular}

\begin{tabular}{|l|l|l|}
\hline Spurions & Operator $(\bar{u} d$ type $)$ & Explicit expression in flavour components \\
\hline$V^{0}$ & $\alpha_{1} \bar{u}_{3} d_{3}+$ h.c. & $\alpha_{1}\left(\bar{u}_{3} d_{3}\right)+$ h.c. \\
$V^{1}, V^{2}, \Delta^{1}$ & - & \\
$\Delta^{1} V^{1}$ & $\sigma_{1} \bar{U} \Delta_{u}^{\dagger} V_{q} d_{3}+$ h.c. & $\approx \sigma_{1} \epsilon_{q}\left[\delta_{u}\left(\bar{u}_{2} d_{3}\right)+s_{u} e^{i \alpha_{u}} \delta_{u}^{\prime}\left(\bar{u}_{1} d_{3}\right)\right]+$ h.c. \\
$\Delta^{1} V^{1}$ & $\sigma_{2} \bar{u}_{3} V_{q}^{\dagger} \Delta_{d} D+$ h.c. & $\approx \sigma_{2} \epsilon_{q}\left[\delta_{d}\left(\bar{u}_{3} d_{2}\right)+s_{d} e^{-i \alpha_{d}} \delta_{d}^{\prime}\left(\bar{u}_{3} d_{1}\right)\right]+$ h.c. \\
\hline
\end{tabular}

Table 3. Right-handed fermion bilinears allowed by different U(2) breaking terms. Notation as in table 2.

Expressing the result via the flavour tensor $\Lambda_{L R}$ we find

$$
\bar{\ell}_{p} \Gamma \Lambda_{L R}^{p r} e_{r}, \quad \Lambda_{L R}=\left(\begin{array}{ccc}
\rho_{1} \delta_{e}^{\prime} & -\rho_{1} s_{e} \delta_{e} & 0 \\
\rho_{1} s_{e} \delta_{e}^{\prime} & \rho_{1} \delta_{e} & \beta_{1} \epsilon_{\ell} \\
\sigma_{1} \epsilon_{\ell} s_{e} \delta_{e}^{\prime} & \sigma_{1} \epsilon_{\ell} \delta_{e} & \alpha_{1}
\end{array}\right)+\mathcal{O}\left(\delta_{e} \epsilon_{\ell}^{2}\right)
$$

Terminating the expansion up to $\mathcal{O}(\Delta V)$, we find 4 complex coefficients, to be compared with the potential 9 complex coefficients in absence of any flavour symmetry. For the same argument discussed in the case of the right-handed structures, in this case a span of the entire flavour space require terms with up to three powers of $\Delta$.

Summary. The total number of CP-even and CP-odd coefficients for all the operators with fermion bilinears constructed with spurions up to $\mathcal{O}\left(\Delta^{1} V^{1}\right)$ are reported in table 5 .

\subsection{Four fermion operators}

In this section we proceed analysing the operators in class 8 which contain four fermion fields. In analogy to the 2-index tensors $\Lambda$ introduced to describe the fermion bilinears, the flavour structure of theses operators is described by 4 -index tensors $\Sigma$. As an illustration, and also in view of the phenomenological application in section 5 , in the case of $(\bar{L} L)(\bar{L} L)$ operators we present the explicit component structure of these tensor. For the other operators we simply list the allowed structures up to $\mathcal{O}\left(V^{3}, \Delta^{1} V^{1}\right)$. 


\begin{tabular}{|l|l|l|}
\hline Spurions & Operator & Explicit expression in flavour components \\
\hline$V^{0}$ & $\alpha_{1} \bar{\ell}_{3} e_{3}$ & $\alpha_{1}\left(\bar{\ell}_{3} e_{3}\right)$ \\
$V^{1}$ & $\beta_{1} \bar{L} V_{\ell} e_{3}$ & $\beta_{1} \epsilon_{\ell}\left(\bar{\ell}_{2} e_{3}\right)$ \\
$V^{2}$ & - & \\
$\Delta^{1}$ & $\rho_{1} \bar{L} \Delta_{e} E$ & $\approx \rho_{1}\left[\delta_{e}\left(\bar{\ell}_{2} e_{2}\right)-s_{e} \delta_{e}\left(\bar{\ell}_{1} e_{2}\right)+s_{e} \delta_{e}^{\prime}\left(\bar{\ell}_{2} e_{1}\right)+\delta_{e}^{\prime}\left(\bar{\ell}_{1} e_{1}\right)\right]$ \\
$\Delta^{1} V^{1}$ & $\sigma_{1} \bar{\ell}_{3} V_{\ell}^{\dagger} \Delta_{e} E$ & $\approx \sigma_{1} \epsilon_{\ell}\left[\delta_{e}\left(\bar{\ell}_{3} e_{2}\right)+s_{e} \delta_{e}^{\prime}\left(\bar{\ell}_{3} e_{1}\right)\right]$ \\
\hline
\end{tabular}

Table 4. Left-right fermion bilinears allowed by different $\mathrm{U}(2)$ breaking terms (the sum over hermitian conjugates is understood for all structures). Notation as in table 2.

\begin{tabular}{|l|c|cc|cc|cc|cc|c|c|}
\hline & N. indep. & \multicolumn{8}{|c|}{$\mathrm{U}(2)^{5}$} & breaking terms \\
Class & structures & \multicolumn{2}{|c|}{$V^{0}$} & \multicolumn{2}{|c|}{$V^{1}$} & $V^{2}$ & $\Delta^{1}$ & $\Delta^{1} V^{1}$ \\
\hline $5 \&$ \& $(\bar{L} R)$ & 11 & 11 & 11 & 11 & 11 & - & - & 11 & 11 & 11 & 11 \\
$7:(\bar{L} L)$ & 4 & 8 & - & 4 & 4 & 4 & - & - & - & - & - \\
$7:(\bar{R} R)$ & 3 & 6 & - & - & - & - & - & - & - & 3 & 3 \\
$7: Q_{\text {Hud }}$ & 1 & 1 & 1 & - & - & - & - & - & - & 2 & 2 \\
\hline total: & 19 & 26 & 12 & 15 & 15 & 4 & - & 11 & 11 & 16 & 16 \\
\hline
\end{tabular}

Table 5. Number of independent operators with fermion bilinears in $\mathrm{U}(2)^{5}$. Notation as in table 1; however, here each column denotes the operators with a precise power of spurions, as indicated in the first row.

$(\overline{\boldsymbol{L}} \boldsymbol{L})(\overline{\boldsymbol{L}} \boldsymbol{L})$ structures. In this category of operators we can distinguish two different subclasses as far as flavour structure and spurion analysis are concerned. The first one contains operators where both bilinears are of the same form, namely $Q_{\ell \ell}, Q_{q q}^{(1)}$ and $Q_{q q}^{(3)}$. Considering $Q_{\ell \ell}$ as representative example of this class of operators, the terms generated up to $\mathcal{O}\left(V^{3}\right)$ are

$$
\begin{aligned}
V^{0}: & {\left[a_{1}\left(\bar{L}^{p} L^{p}\right)\left(\bar{L}^{r} L^{r}\right)+a_{2}\left(\bar{L}^{p} L^{r}\right)\left(\bar{L}^{r} L^{p}\right)+a_{3}(\bar{L} L)\left(\bar{\ell}_{3} \ell_{3}\right)\right.} \\
& \left.+a_{4}\left(\bar{L} \ell_{3}\right)\left(\bar{\ell}_{3} L\right)+a_{5}\left(\bar{\ell}_{3} \ell_{3}\right)\left(\bar{\ell}_{3} \ell_{3}\right)\right], \\
V^{1}: & {\left[\beta_{1}\left(\bar{L}^{p} V_{\ell}^{p} \ell_{3}\right)\left(\bar{L}^{r} L^{r}\right)+\beta_{2}\left(\bar{L} V_{\ell} \ell_{3}\right)\left(\bar{\ell}_{3} \ell_{3}\right)+\beta_{3}\left(\bar{L}^{p} V_{\ell}^{p} L^{r}\right)\left(\bar{L}^{r} \ell_{3}\right)+\text { h.c. }\right], } \\
V^{2}: & {\left[c_{1}\left(\bar{L}^{p} V_{\ell}^{p} V_{\ell}^{\dagger r} L^{r}\right)\left(\bar{L}^{s} L^{s}\right)+c_{2}\left(\bar{L}^{p} V_{\ell}^{p} V_{\ell}^{\dagger r} L^{r}\right)\left(\bar{\ell}_{3} \ell_{3}\right)+c_{3}\left(\bar{L}^{p} V_{\ell}^{p} \ell_{3}\right)\left(\bar{\ell}_{3} V_{\ell}^{\dagger r} L^{r}\right)\right.} \\
& \left.+c_{4}\left(\bar{L}^{p} V_{\ell}^{p} L^{r}\right)\left(\bar{L}^{r} V_{\ell}^{\dagger s} L^{s}\right)+\left(\gamma_{1}\left(\bar{L}^{p} V_{\ell}^{p} \ell_{3}\right)\left(\bar{L}^{r} V_{\ell}^{r} \ell_{3}\right)+\text { h.c. }\right)\right], \\
V^{3}: & {\left[\xi_{1}\left(\bar{L}^{p} V_{\ell}^{p} V_{\ell}^{\dagger r} L^{r}\right)\left(\bar{L}^{s} V_{\ell}^{s} \ell_{3}\right)+\text { h.c. }\right] . }
\end{aligned}
$$


For the remaining two operators, $Q_{\ell q}^{(1)}$ and $Q_{\ell q}^{(3)}$, we get the following terms:

$$
\begin{aligned}
V^{0}: & {\left[a_{1}(\bar{L} L)(\bar{Q} Q)+a_{2}(\bar{L} L)\left(\bar{q}_{3} q_{3}\right)+a_{3}\left(\bar{\ell}_{3} \ell_{3}\right)(\bar{Q} Q)+a_{4}\left(\bar{\ell}_{3} \ell_{3}\right)\left(\bar{q}_{3} q_{3}\right)\right], } \\
V^{1}: & {\left[\beta_{1}\left(\bar{L} V_{\ell} \ell_{3}\right)(\bar{Q} Q)+\beta_{2}\left(\bar{L} V_{\ell} \ell_{3}\right)\left(\bar{q}_{3} q_{3}\right)+\beta_{3}(\bar{L} L)\left(\bar{Q} V_{q} q_{3}\right)+\beta_{4}\left(\bar{\ell}_{3} \ell_{3}\right)\left(\bar{Q} V_{q} q_{3}\right)+\text { h.c. }\right], } \\
V^{2}: & {\left[c_{1}\left(\bar{L}^{p} V_{\ell}^{p} V_{\ell}^{\dagger r} L^{r}\right)(\bar{Q} Q)+c_{2}\left(\bar{L}^{p} V_{\ell}^{p} V_{\ell}^{\dagger r} L^{r}\right)\left(\bar{q}_{3} q_{3}\right)+c_{3}(\bar{L} L)\left(\bar{Q}^{p} V_{q}^{p} V_{q}^{\dagger r} Q^{r}\right)\right.} \\
& \left.+c_{4}\left(\bar{\ell}_{3} \ell_{3}\right)\left(\bar{Q}^{p} V_{q}^{p} V_{q}^{\dagger r} Q^{r}\right)+\left(\gamma_{1}\left(\bar{L} V_{\ell} \ell_{3}\right)\left(\bar{Q} V_{q} q_{3}\right)+\gamma_{2}\left(\bar{L} V_{\ell} \ell_{3}\right)\left(\bar{q}_{3} V_{q}^{\dagger} Q\right)+\text { h.c. }\right)\right], \\
V^{3}: & {\left[\xi_{1}\left(\bar{L}^{p} V_{\ell}^{p} V_{\ell}^{\dagger r} L^{r}\right)\left(\bar{Q} V_{q} q_{3}\right)+\xi_{2}\left(\bar{L} V_{\ell} \ell_{3}\right)\left(\bar{Q}^{p} V_{q}^{p} V_{q}^{\dagger r} Q^{r}\right)+\text { h.c. }\right] . }
\end{aligned}
$$

No additional terms arise with the insertion of one power of $\Delta$. On the other hand, it is worth stressing that the $(\bar{L} L)(\bar{L} L)$ operators are the only ones where terms with 3 powers of the $V$ spurions are relevant (more details about the number of independent fermion contractions for four-fermion operators are given in appendix B).

For each electroweak structure of $(\bar{L} L)(\bar{L} L)$ operators we therefore find the following number of real and imaginary coefficients at a given order in the spurion expansion:

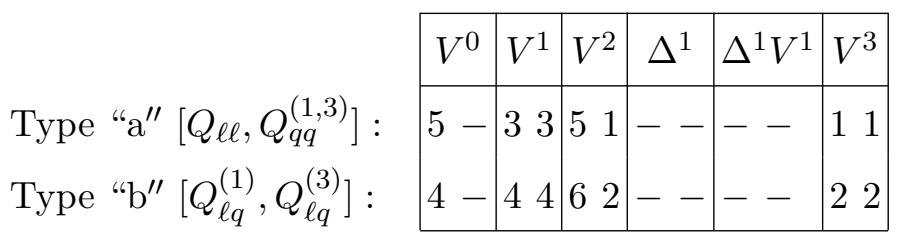

As anticipated, the flavour structure of the four-fermion operators is described by the 4index tensors $\Sigma$. In the specific case of the structures in eqs. (3.13)-(3.14) we defined them as

$$
\Sigma_{\ell \ell}^{i j, n m}\left(\bar{\ell}_{i} \Gamma \ell_{j}\right)\left(\bar{\ell}_{n} \Gamma \ell_{m}\right) \quad \text { and } \quad \Sigma_{\ell q}^{i j, n m}\left(\bar{\ell}_{i} \Gamma \ell_{j}\right)\left(\bar{q}_{n} \Gamma q_{m}\right)
$$

The corresponding explicit expressions are reported in table 12 and 13 in appendix C, respectively.

$(\overline{\boldsymbol{R}} \boldsymbol{R})(\overline{\boldsymbol{R}} \boldsymbol{R})$ structures. In this case we can distinguish three different subclasses of operators. The first one includes operators with identical right-handed quark fields, namely $Q_{u u}$ and $Q_{d d}$. Considering $Q_{u u}$ as representative example of this subclass of operators, the terms generated up to $\mathcal{O}\left(V^{3}, \Delta^{1} V^{1}\right)$ are

$$
\begin{aligned}
V^{0}: \quad & {\left[a_{1}\left(\bar{U}^{p} U^{p}\right)\left(\bar{U}^{r} U^{r}\right)+a_{2}\left(\bar{U}^{p} U^{r}\right)\left(\bar{U}^{r} U^{p}\right)+a_{3}(\bar{U} U)\left(\bar{u}_{3} u_{3}\right)\right.} \\
& \left.+a_{4}\left(\bar{U} u_{3}\right)\left(\bar{u}_{3} U\right)+a_{5}\left(\bar{u}_{3} u_{3}\right)\left(\bar{u}_{3} u_{3}\right)\right], \\
\Delta^{1} V^{1}: & {\left[\sigma_{1}\left(\bar{u}_{3} V_{q}^{\dagger s} \Delta_{u}^{s, r} U^{r}\right)\left(\bar{U}^{p} U^{p}\right)+\sigma_{2}\left(\bar{u}_{3} V_{q}^{\dagger} \Delta_{u} U\right)\left(\bar{u}_{3} u_{3}\right)\right.} \\
& \left.+\sigma_{3}\left(\bar{U}^{p} V_{q}^{\dagger s} \Delta_{u}^{s r} U^{r}\right)\left(\bar{u}_{3} U^{p}\right)+\text { h.c. }\right] .
\end{aligned}
$$

The second type is the operator $Q_{e e}$, which also involves identical right-handed fields. The decomposition proceeds as for $Q_{u u}$; however, due to the Fierz identity in eq. (2.5), we should not consider as independent terms of the type $\left(\bar{E} e_{3}\right)\left(\bar{e}_{3} E\right)$ and $\left(\bar{E}^{p} E^{r}\right)\left(\bar{E}^{r} E^{p}\right)$, which reduce to $(\bar{E} E)\left(\bar{e}_{3} e_{3}\right)$ and $\left(\bar{E}^{p} E^{p}\right)\left(\bar{E}^{r} E^{r}\right)$ respectively. Similarly, at higher order in the spurion expansion, we can relate the operator $\left(V_{q}^{\dagger s} \Delta_{e}^{s, r}\right)\left(\bar{E}^{p} E^{r}\right)\left(\bar{e}_{3} E^{p}\right)$ to $\left(V_{q}^{\dagger s} \Delta_{e}^{s, r}\right)\left(\bar{E}^{p} E^{p}\right)\left(\bar{e}_{3} E^{r}\right)$. 
For the remaining four operators $Q_{e u}, Q_{e d}, Q_{u d}^{(1)}$ and $Q_{u d}^{(8)}$ the counting is the same. ${ }^{7}$ Considering $Q_{e u}$ as representative example of this subclass we find

$$
\begin{aligned}
V^{0}: & {\left[a_{1}(\bar{E} E)(\bar{U} U)+a_{2}(\bar{E} E)\left(\bar{u}_{3} u_{3}\right)+a_{3}\left(\bar{e}_{3} e_{3}\right)(\bar{U} U)+a_{4}\left(\bar{e}_{3} e_{3}\right)\left(\bar{u}_{3} u_{3}\right)\right] } \\
\Delta^{1} V^{1}: \quad & {\left[\sigma_{1}\left(\bar{e}_{3} V_{\ell}^{\dagger} \Delta_{e} E\right)(\bar{U} U)+\sigma_{2}\left(\bar{e}_{3} V_{\ell}^{\dagger} \Delta_{e} E\right)\left(\bar{u}_{3} u_{3}\right)\right.} \\
& \left.+\sigma_{3}(\bar{E} E)\left(\bar{u}_{3} V_{q}^{\dagger} \Delta_{u} U\right)+\sigma_{4}\left(\bar{e}_{3} e_{3}\right)\left(\bar{u}_{3} V_{q}^{\dagger} \Delta_{u} U\right)+\text { h.c. }\right] .
\end{aligned}
$$

For each electroweak structure of $(\bar{R} R)(\bar{R} R)$ operators we therefore find the following number of real and imaginary coefficients at a given order in the spurion expansion:

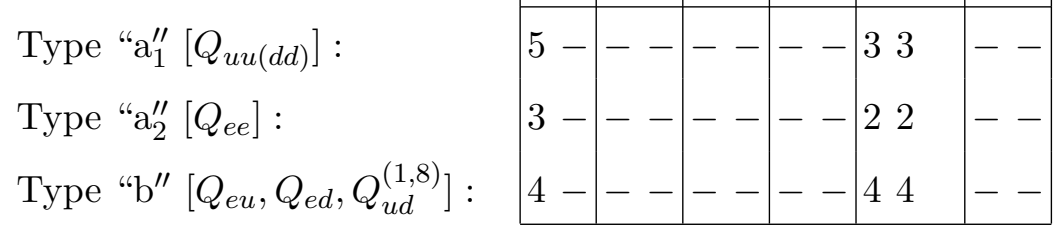

$(\overline{\boldsymbol{L}} \boldsymbol{L})(\overline{\boldsymbol{R}} \boldsymbol{R})$ structures. In this case we distinguish again two sub-classes. The first one includes $Q_{l e}, Q_{q u}^{(1,8)}$, and $Q_{q d}^{(1,8)}$. Considering $Q_{l e}$ as representative example, the terms generated up to $\mathcal{O}\left(V^{3}, \Delta^{1} V^{1}\right)$ are

$$
\begin{aligned}
V^{0}: & {\left[a_{1}(\bar{L} L)(\bar{E} E)+a_{2}(\bar{L} L)\left(\bar{e}_{3} e_{3}\right)+a_{3}\left(\bar{\ell}_{3} \ell_{3}\right)(\bar{E} E)+a_{4}\left(\bar{\ell}_{3} \ell_{3}\right)\left(\bar{e}_{3} e_{3}\right)\right], } \\
V^{1}: & {\left[\beta_{1}\left(\bar{L} V_{\ell} \ell_{3}\right)(\bar{E} E)+\beta_{2}\left(\bar{L} V_{\ell} \ell_{3}\right)\left(\bar{e}_{3} e_{3}\right)+\text { h.c. }\right], } \\
V^{2}: & {\left[c_{1}\left(\bar{L}^{p} V_{\ell}^{p} V_{\ell}^{\dagger r} L^{r}\right)(\bar{E} E)+c_{2}\left(\bar{L}^{p} V_{\ell}^{p} V_{\ell}^{\dagger r} L^{r}\right)\left(\bar{e}_{3} e_{3}\right)\right], } \\
\Delta^{1} V^{0}: & {\left[\rho_{1}\left(\bar{L} \ell_{3}\right) \Delta_{e}\left(\bar{e}_{3} E\right)+\text { h.c. }\right], } \\
\Delta^{1} V^{1}: & {\left[\sigma_{1}\left(\bar{L}^{p} V_{\ell}^{\dagger r} L^{r}\right) \Delta_{e}^{p t}\left(\bar{e}_{3} E^{t}\right)+\sigma_{2}\left(\bar{L}^{p} L^{p}\right) V_{\ell}^{\dagger r} \Delta_{e}^{r t}\left(\bar{e}_{3} E^{t}\right)\right.} \\
& \left.+\sigma_{3}\left(\bar{\ell}_{3} \ell_{3}\right) V_{\ell}^{\dagger} \Delta_{e}\left(\bar{e}_{3} E\right)+\text { h.c. }\right] .
\end{aligned}
$$

For the remaining operators $Q_{l u}, Q_{l d}$, and $Q_{q e}$, considering $Q_{l u}$ as representative, we get

$$
\begin{aligned}
V^{0}: & {\left[a_{1}(\bar{L} L)(\bar{U} U)+a_{2}(\bar{L} L)\left(\bar{u}_{3} u_{3}\right)+a_{3}\left(\bar{\ell}_{3} \ell_{3}\right)(\bar{U} U)+a_{4}\left(\bar{\ell}_{3} \ell_{3}\right)\left(\bar{u}_{3} u_{3}\right)\right], } \\
V^{1}: & {\left[\beta_{1}\left(\bar{L} V_{\ell} \ell_{3}\right)(\bar{U} U)+\beta_{2}\left(\bar{L} V_{\ell} \ell_{3}\right)\left(\bar{u}_{3} u_{3}\right)+\text { h.c. }\right] } \\
V^{2}: & {\left[c_{1}\left(\bar{L}^{p} V_{\ell}^{p} V_{\ell}^{\dagger r} L^{r}\right)(\bar{U} U)+c_{2}\left(\bar{L}^{p} V_{\ell}^{p} V_{\ell}^{\dagger r} L^{r}\right)\left(\bar{u}_{3} u_{3}\right)\right], } \\
\Delta^{1} V^{1}: & {\left[\sigma_{1}(\bar{L} L) V_{q}^{\dagger} \Delta_{u}\left(\bar{u}_{3} U\right)+\sigma_{2}\left(\bar{\ell}_{3} \ell_{3}\right) V_{q}^{\dagger} \Delta_{u}\left(\bar{u}_{3} U\right)+\text { h.c. }\right] . }
\end{aligned}
$$

For each electroweak structure of $(\bar{L} L)(\bar{R} R)$ operators we therefore find the following number of real and imaginary coefficients at a given order in the spurion expansion:

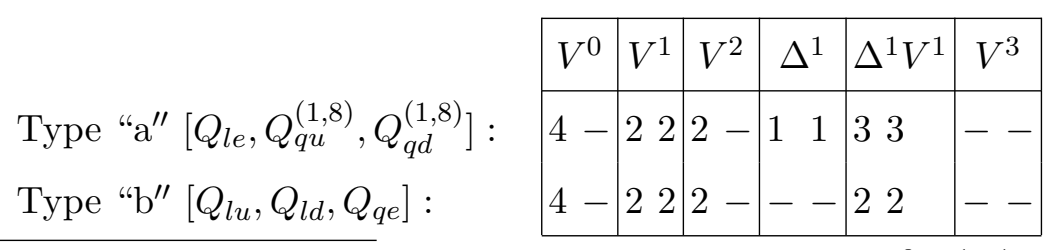

\footnotetext{
${ }^{7}$ This statement holds because we truncate the spurion expansion up to $\mathcal{O}\left(V^{3}, \Delta^{1} V^{1}\right)$ : at higher orders the counting for $Q_{e u}$ and $Q_{e d}$ would start to differ from the counting for $Q_{u d}^{(1)}$ and $Q_{u d}^{(8)}$.
} 
$(\overline{\boldsymbol{L}} \boldsymbol{R})(\overline{\boldsymbol{R}} \boldsymbol{L})$ and $(\overline{\boldsymbol{L}} \boldsymbol{R})(\overline{\boldsymbol{L}} \boldsymbol{R})$ structures. There is a single $(\bar{L} R)(\bar{R} L)$ operator, $Q_{\text {ledq }}$, for which the spurion decomposition up to $\mathcal{O}\left(V^{3}, \Delta^{1} V^{1}\right)$ yields:

$$
\begin{aligned}
V^{0}: & {\left[\alpha_{1}\left(\bar{\ell}_{3} e_{3}\right)\left(\bar{d}_{3} q_{3}\right)+\text { h.c. }\right], } \\
V^{1}: & {\left[\beta_{1}\left(\bar{L} V_{\ell} e_{3}\right)\left(\bar{d}_{3} q_{3}\right)+\beta_{2}\left(\bar{\ell}_{3} e_{3}\right)\left(\bar{d}_{3} V_{q}^{\dagger} Q\right)+\text { h.c. }\right], } \\
V^{2}: & {\left[\gamma_{1}\left(\bar{L} V_{\ell} e_{3}\right)\left(\bar{d}_{3} V_{q}^{\dagger} Q\right)+\text { h.c. }\right] } \\
\Delta^{1} V^{0}: & {\left[\rho_{1}\left(\bar{L} \Delta_{e} E\right)\left(\bar{d}_{3} q_{3}\right)+\rho_{2}\left(\bar{\ell}_{3} e_{3}\right)\left(\bar{D} \Delta_{d}^{\dagger} Q\right)+\text { h.c. }\right], } \\
\Delta^{1} V^{1}: & {\left[\sigma_{1}\left(\bar{L} \Delta_{e} E\right)\left(\bar{d}_{3} V_{q}^{\dagger} Q\right)+\sigma_{2}\left(\bar{\ell}_{3} V_{\ell}^{\dagger} \Delta_{e} E\right)\left(\bar{d}_{3} q_{3}\right)\right.} \\
+ & \left.+\sigma_{3}\left(\bar{L} V_{\ell} e_{3}\right)\left(\bar{D} \Delta_{d}^{\dagger} Q\right)+\sigma_{4}\left(\bar{\ell}_{3} e_{3}\right)\left(\bar{D} \Delta_{d}^{\dagger} V_{q} q_{3}\right)+\text { h.c. }\right],
\end{aligned}
$$

As far as $(\bar{L} R)(\bar{L} R)$ structures are concerned, we need to distinguish between $Q_{\text {lequ }}^{(1,3)}$ and $Q_{q u q d}^{(1,3)}$. In the first case we have the same decomposition as for $Q_{\text {ledq }}$, while in the second case we get

$$
\begin{aligned}
V^{0}: & {\left[\alpha_{1}\left(\bar{q}_{3} u_{3}\right)\left(\bar{q}_{3} d_{3}\right)+\text { h.c. }\right] } \\
V^{1}: & {\left[\beta_{1}\left(\bar{Q} V_{q} u_{3}\right)\left(\bar{q}_{3} d_{3}\right)+\beta_{2}\left(\bar{q}_{3} u_{3}\right)\left(\bar{Q} V_{q} d\right)+\text { h.c. }\right] } \\
V^{2}: & {\left[\gamma_{1}\left(\bar{Q} V_{q} u_{3}\right)\left(\bar{Q} V_{q} d_{3}\right)+\text { h.c. }\right] } \\
\Delta^{1} V^{0}: & {\left[\rho_{1}\left(\bar{Q} \Delta_{u} U\right)\left(\bar{q}_{3} d_{3}\right)+\rho_{2}\left(\bar{q}_{3} u_{3}\right)\left(\bar{Q} \Delta_{d} D\right)\right.} \\
& \left.+\rho_{3}\left(\bar{q}_{3} \Delta_{u} U\right)\left(\bar{Q} d_{3}\right)+\rho_{4}\left(\bar{Q} u_{3}\right)\left(\bar{q}_{3} \Delta_{d} D\right)+\text { h.c. }\right] \\
\Delta^{1} V^{1}: & {\left[\sigma_{1}\left(\bar{q}_{3} V_{q}^{\dagger} \Delta_{u} U\right)\left(\bar{q}_{3} d_{3}\right)+\sigma_{2}\left(\bar{Q}^{p} \Delta_{u}^{p r} U^{r}\right)\left(\bar{Q}^{s} V_{q}^{s} d_{3}\right)+\sigma_{3}\left(\bar{q}_{3} u_{3}\right)\left(\bar{q}_{3} V_{q}^{\dagger} \Delta_{d} D\right)\right.} \\
& +\sigma_{4}\left(\bar{Q}^{p} V_{q}^{p} u_{3}\right)\left(\bar{Q}^{r} \Delta_{d}^{r s} D^{s}\right)+\sigma_{5}\left(\bar{Q}^{p} V_{q}^{p} U^{r}\right) \Delta_{u}^{s r}\left(\bar{Q}^{s} d_{3}\right)+\sigma_{6}\left(\bar{Q}^{p} u_{3}\right) \Delta_{d}^{p r}\left(\bar{Q}^{s} V_{q}^{s} D^{r}\right) \\
+ & \text { h.c. }] .
\end{aligned}
$$

For each electroweak structure of $(\bar{L} R)(\bar{R} L)$ and $(\bar{L} R)(\bar{L} R)$ operators we therefore find the following number of real and imaginary coefficients at a given order in the spurion expansion:

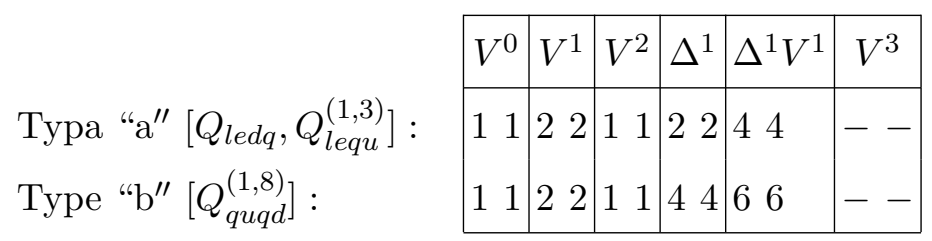

\subsection{Summary and discussion}

The results for all SMEFT operators are summarized in table 6, while the detailed counting order by order, organised according to the different sub-categories of operators is presented in table 10 in appendix C. As expected, the smaller symmetry group leads to a significantly larger number of terms compared to the MFV case in table 1. However, we emphasise that the number of independent terms is still rather small compared to the case of no symmetry, even when considering high powers of the spurions. It is also worth stressing that the smallness (and the nature) of the $\mathrm{U}(2)^{5}$ breaking terms allows us to consider only limited subsets of the terms reported in table 6 depending on the observables, and the level of precision, we are interested in. For instance, in the limit where we neglect the masses of the first two generations (which is often an excellent approximation) we can stop at the third column. 


\begin{tabular}{|l|ll|ll|ll|l|ll|ll|ll|l|l|l|}
\hline & \multicolumn{10}{|c|}{$\mathrm{U}(2)^{5}$ [terms summed up to different orders] } \\
Operators & Exact & $\mathcal{O}\left(V^{1}\right)$ & $\mathcal{O}\left(V^{2}\right)$ & $\mathcal{O}\left(V^{1}, \Delta^{1}\right)$ & $\mathcal{O}\left(V^{2}, \Delta^{1}\right)$ & $\mathcal{O}\left(V^{2}, \Delta^{1} V^{1}\right)$ & $\mathcal{O}\left(V^{3}, \Delta^{1} V^{1}\right)$ \\
\hline Class 1-4 & 9 & 6 & 9 & 6 & 9 & 6 & 9 & 6 & 9 & 6 & 9 & 6 & 9 & 6 \\
\hline$\psi^{2} H^{3}$ & 3 & 3 & 6 & 6 & 6 & 6 & 9 & 9 & 9 & 9 & 12 & 12 & 12 & 12 \\
$\psi^{2} X H$ & 8 & 8 & 16 & 16 & 16 & 16 & 24 & 24 & 24 & 24 & 32 & 32 & 32 & 32 \\
$\psi^{2} H^{2} D$ & 15 & 1 & 19 & 5 & 23 & 5 & 19 & 5 & 23 & 5 & 28 & 10 & 28 & 10 \\
\hline$(\bar{L} L)(\bar{L} L)$ & 23 & - & 40 & 17 & 67 & 24 & 40 & 17 & 67 & 24 & 67 & 24 & 74 & 31 \\
$(\bar{R} R)(\bar{R} R)$ & 29 & - & 29 & - & 29 & - & 29 & - & 29 & - & 53 & 24 & 53 & 24 \\
$(\bar{L} L)(\bar{R} R)$ & 32 & - & 48 & 16 & 64 & 16 & 53 & 21 & 69 & 21 & 90 & 42 & 90 & 42 \\
$(\bar{L} R)(\bar{R} L)$ & 1 & 1 & 3 & 3 & 4 & 4 & 5 & 5 & 6 & 6 & 10 & 10 & 10 & 10 \\
$(\bar{L} R)(\bar{L} R)$ & 4 & 4 & 12 & 12 & 16 & 16 & 24 & 24 & 28 & 28 & 48 & 48 & 48 & 48 \\
\hline total: & 124 & 23 & 182 & 81 & 234 & 93 & 212 & 111 & 264 & 123 & 349 & 208 & 356 & 215 \\
\hline
\end{tabular}

Table 6. Number of independent operators in the SMEFT assuming a minimally broken $\mathrm{U}(2)^{5}$ symmetry, including breaking terms up to $\mathcal{O}\left(V^{3}, \Delta^{1} V^{1}\right)$. Notations as in table 1.

\section{Beyond $\mathrm{U}(3)^{5}$ and $\mathrm{U}(2)^{5}$ with minimal breaking}

The main virtue of the MFV hypothesis is to normalise the magnitude of flavour-violating processes in the quark sector to their corresponding size in the SM (controlled by the CKM matrix), both for tree-level amplitudes and for the leading loop-induced ones. This is achieved by linking every possible source of flavour non-universality to the Yukawa couplings. Beside this rather strong assumption from the model-building point of view, the MFV hypothesis has two main drawbacks in the EFT implementation: i) no clear powercounting due to the large value of $y_{t}$ (hence no special role for the third generation which, on the other hand, plays a key role in the Higgs hierarchy problem); ii) no flavour mixing in the lepton sector. The advantage of the $\mathrm{U}(2)^{5}$ setup, with the minimal breaking discussed in section 3, is that it addresses these two drawbacks preserving the main virtue of the MFV setup in flavour-violating processes. Moreover, this goal is achieved without imposing a specific alignment among the spurions, but only a well-defined hierarchical structure. The price to pay is a significant enlargement in the number of free parameters due to the smaller symmetry group. In this section we briefly discuss if it is worth to consider alternative options, either as far as flavour symmetries or as far as symmetry-breaking terms are concerned.

\subsection{Unbroken $\mathrm{U}(3)_{d, e}$ groups}

A first natural question to ask is if we can consider an intermediate case, combining $\mathrm{U}(2)$ groups in the up-quark sector and $\mathrm{U}(3)$ subgroups in the right-handed down-quark and/or charged-lepton sector, as for instance recently proposed in [28]. To clarify this point, let's consider the breaking chain in the quark sector due to the third-generation Yukawa couplings. Similarly to eq. (2.11), if we consider a two-step breaking due to $y_{t}$ and, later on by $y_{b}$ (that eventually we are interested to treat as a spurion), the impact on the whole 
quark-flavour symmetry group is $[26,27]^{8}$

$$
\mathrm{U}(3)_{q} \otimes \mathrm{U}(3)_{u} \otimes \mathrm{U}(3)_{d} \stackrel{y_{t}}{\longrightarrow} \mathrm{U}(2)_{q} \otimes \mathrm{U}(2)_{u} \otimes \mathrm{U}(3)_{d} \otimes \mathrm{U}(1)_{3}^{\prime} \stackrel{y_{t, b}}{\longrightarrow} \mathrm{U}(2)_{q} \otimes \mathrm{U}(2)_{u} \otimes \mathrm{U}(2)_{d} \otimes \mathrm{U}(1)_{3}^{\prime \prime} .
$$

As can be seen, in the first step we are left with an unbroken $\mathrm{U}(3)$ subgroup. However, this case is less appealing than the $\mathrm{U}(2)^{5}$ minimal setup, since we would need to impose a spefic alignment among the spurions to recover a MFV-like suppression of flavour-changing amplitudes. This follows from the decompositon of the SM Yukawa couplings in terms of spurions of the unbroken subgroups in the two cases:

$$
\begin{aligned}
& Y_{u, d} \stackrel{y_{t}}{\longrightarrow} V_{q} \oplus \Delta_{u} \oplus\left[\Lambda_{d} \sim(1,1, \overline{3}) \oplus \Sigma_{d} \sim(2,1, \overline{3}) \oplus V_{R}^{u} \sim(1, \overline{2}, 1)\right]_{\mathrm{U}(2)_{q} \otimes \mathrm{U}(2)_{u} \otimes \mathrm{U}(3)_{d}}, \\
& Y_{u, d} \stackrel{y_{t, b}}{\longrightarrow} V_{q} \oplus \Delta_{u} \oplus \Delta_{d} \oplus\left[V_{R}^{u} \sim(1, \overline{2}, 1) \oplus V_{R}^{d} \sim(1,1, \overline{2})\right]_{\mathrm{U}(2)^{3}} .
\end{aligned}
$$

Here $V_{q}$ and $\Delta_{u, d}$ denote the spurions that we have in the $\mathrm{U}(2)^{5}$ minimal setup, whereas between brackets we show the additional terms arising in the decomposition.

I No unbroken $\mathrm{U}(3)$ subgroups, eq. (4.3). Here we simply need to assume the hierarchy $\left|V_{q}\right| \gg\left|\Delta_{u, d}\right| \gg\left|V_{R}^{u, d}\right|$ to recover a MFV-like structure and, at the same time, have all terms necessary to reconstruct completely the SM Yukawa couplings.

II Unbroken $\mathrm{U}(3)_{d}$ group, eq. (4.2). Here we cannot neglect the terms between brackets if we wish to describe down-quark masses. Allowing only $\Lambda_{d} \neq 0$ we can describe $y_{b} \neq 0$, but strange and down quark remain massless. A description of all the masses require both $\Lambda_{d} \neq 0$ and $\Sigma_{d} \neq 0$, with a necessary tuning of their alignment in the $\mathrm{U}(3)_{d}$ space in order to avoid large right-handed mixing (which is strongly constrained by data).

In view of these considerations, we consider the $\mathrm{U}(2)^{5}$ case more interesting, from a model-building point of view, with respect to a framework with some unbroken U(3) subgroups [28]. The only variation that is worth to consider, in order to treat $y_{b}$ and/or $y_{\tau}$ as small parameters, is the addition of appropriate $\mathrm{U}(1)$ groups acting only on the right-handed $b$ and $\tau$ fields, that is what we discuss next.

\section{$4.2 \quad \mathrm{U}(2)^{5} \otimes \mathrm{U}(1)_{b} \otimes \mathrm{U}(1)_{\tau}$}

Enlarging the $\mathrm{U}(2)^{5}$ symmetry with two $\mathrm{U}(1)$ groups under which only $b_{R}$ and $\tau_{R}$ are charged, the only mass term allowed in the limit of unbroken symmetry is the top Yukawa coupling. In order to allow non-vanishing bottom and tau Yukawa couplings, two spurions $X_{b(\tau)}$, breaking the $\mathrm{U}(1)_{b(\tau)}$ groups, must be included. Considering these spurions, in addition to those of the minimal setup, the Yukawa matrices assume the form

$$
Y_{u}=y_{t}\left(\begin{array}{cc}
\Delta_{u} & x_{t} V_{q} \\
0 & 1
\end{array}\right), \quad Y_{d}=\left(\begin{array}{cc}
\Delta_{d}^{\prime} & x_{b}^{\prime} X_{b} V_{q} \\
0 & \kappa_{b} X_{b}
\end{array}\right), \quad Y_{e}=\left(\begin{array}{cc}
\Delta_{e}^{\prime} & x_{\tau}^{\prime} X_{\tau} V_{\ell} \\
0 & \kappa_{\tau} X_{\tau}
\end{array}\right)
$$

\footnotetext{
${ }^{8}$ Here $\mathrm{U}(1)_{3}^{\prime}=\mathrm{U}(1)_{q_{L}^{3}+t_{R}}$ and $\mathrm{U}(1)_{3}^{\prime \prime}=\mathrm{U}(1)_{q_{L}^{3}+b_{R}+t_{R}}$.
} 


\begin{tabular}{|c|c|c|c|c|c|c|c|c|c|c|c|c|c|c|c|}
\hline \multirow{3}{*}{$\begin{array}{l}\text { Operators } \\
\text { Class } 1-4 \\
\end{array}$} & \multicolumn{15}{|c|}{$\mathrm{U}(2)^{5} \otimes \mathrm{U}(1)_{b} \otimes \mathrm{U}(1)_{\tau}$ [terms summed up to different orders] } \\
\hline & \multirow{2}{*}{\begin{tabular}{|l} 
Exact \\
96
\end{tabular}} & \multicolumn{2}{|c|}{$\mathcal{O}\left(X^{2}\right)$} & \multicolumn{2}{|c|}{$\mathcal{O}\left(V^{1}, X^{2}\right)$} & \multicolumn{2}{|c|}{$\mathcal{O}\left(V^{2}, V^{1} X^{2}\right)$} & \multicolumn{2}{|c|}{$\mathcal{O}\left(\Delta^{1}, V^{1} X^{2}\right)$} & \multicolumn{2}{|c|}{$\begin{array}{r}\mathcal{O}\left(V^{2}, \Delta^{1},\right. \\
\left.V^{1} X^{2}\right)\end{array}$} & \multicolumn{2}{|c|}{$\mid \begin{array}{c}\mathcal{O}\left(\Delta^{1} V^{1}\right. \\
\left.V^{2} X^{2}, \Delta^{1} X^{1}\right)\end{array}$} & \multicolumn{2}{|c|}{$\begin{array}{c}\mathcal{O}\left(V^{3}, \Delta^{1} V^{1}\right. \\
\left.V^{2} X^{2}, \Delta^{1} X^{1}\right)\end{array}$} \\
\hline & & 9 & 6 & 9 & 6 & 9 & 6 & 9 & 6 & 9 & 6 & 9 & 6 & 9 & 6 \\
\hline$\psi^{2} H^{3}$ & 11 & 3 & 3 & 4 & 4 & 6 & 6 & 9 & 9 & 9 & 9 & 12 & 12 & 12 & 12 \\
\hline$\psi^{2} X H$ & 33 & 8 & 8 & 11 & 11 & 16 & 16 & 24 & 24 & 24 & 24 & 32 & 32 & 32 & 32 \\
\hline$\psi^{2} H^{2} D$ & 14 & 15 & 1 & 19 & 5 & 23 & 5 & 19 & 5 & 23 & 5 & 25 & 7 & 25 & 7 \\
\hline$(\bar{L} L)(\bar{L} L)$ & 23 & 23 & 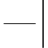 & 40 & 17 & 67 & 24 & 40 & 17 & 67 & 24 & 67 & 24 & 74 & 31 \\
\hline$(\bar{R} R)(\bar{R} R)$ & 29 & 29 & . & 29 & - & 29 & - & 29 & - & 29 & - & 38 & 9 & 38 & 9 \\
\hline$(\bar{L} L)(\bar{R} R)$ & 32 & 32 & 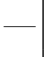 & 48 & 16 & 64 & 16 & 50 & 18 & 66 & 18 & 77 & 29 & 77 & 29 \\
\hline$(\bar{L} R)(\bar{R} L)$ & - & 1 & 1 & 1 & 1 & 3 & 3 & 3 & 3 & 3 & 3 & 6 & 6 & 6 & 6 \\
\hline$(\bar{L} R)(\bar{L} R)$ & - & 4 & 4 & 4 & 4 & 12 & 12 & 18 & 18 & 18 & 18 & 38 & 38 & 38 & 38 \\
\hline total: & 11110 & 124 & 23 & 165 & 64 & 229 & 88 & 201 & 100 & 248 & 107 & 304 & 163 & 311 & 170 \\
\hline
\end{tabular}

Table 7. Number of independent operators at different orders in the spurion expansion for the SMEFT with $\mathrm{U}(2)^{5} \otimes \mathrm{U}(1)^{2}$ flavour symmetry.

where $\Delta_{b(\tau)}^{\prime}$ differ by $\Delta_{b(\tau)}$ only by the overall normalization, and $\kappa_{b, \tau}$ are two additional free parameters that we are allowed to include in this framework, depending on the normalization of the U(1)-breaking terms. The counting of independent coefficients of SMEFT operators at each order in the spurion expansion in this setup, is reported in table 7 , while the detailed counting order by order, organised according to the different sub-categories of operators is presented in table 11 in appendix C. As can be expected, in absence of $X$ spurions, in this framework one finds less terms than at corresponding order in the $V$ and $\Delta$ expansion in the minimal $\mathrm{U}(2)^{5}$ setup. On the other hand, when going to $\mathcal{O}\left(X^{2}\right)$ one recovers the same numbers of independent terms of the minimal $\mathrm{U}(2)^{5}$ setup at the corresponding order in the $V$ and $\Delta$ expansion.

\subsection{Classification of all possible $\mathrm{U}(2)^{5}$ breaking terms}

To conclude this section, we present a list of the possible breaking terms of $\mathrm{U}(2)^{5}$ beyond the minimal setup. The $\mathrm{U}(2)^{5}$ breaking spurions can be easily classified by analysing how the bilinears $\bar{\psi} \psi$ and the four-fermion operators $\bar{\psi} \psi \bar{\psi} \psi$ transform. Focusing on the bilinears only, ${ }^{9} 20$ different structure arise:

- 5 spurions $V_{\psi} \sim\left(\bar{\psi} \psi_{3}\right) \sim 2_{\psi}$, where $\psi=\{L, Q, E, U, D\}$ and $\psi_{3}=\left\{\ell_{3}, q_{3}, \tau_{R}, t_{R}, b_{R}\right\}$ (in the minimal setup only the two $V_{\psi}$ breaking the two left-handed subgroups are included);

- 10 bi-fundamental spurions $\Delta_{\psi \psi^{\prime}} \sim\left(\bar{\psi} \psi^{\prime}\right) \sim \overline{2}_{\psi} \times 2_{\psi^{\prime}}$, with $\psi \neq \psi^{\prime}$, which include 6 leptoquark spurions, 3 di-quark spurions, and 1 di-lepton spurion (in the minimal setup only the three Yukawa-like $\Delta_{\psi \psi^{\prime}}$ are included);

- 5 adjoints spurions $A_{\psi} \sim(\bar{\psi} \psi) \sim 3_{\psi}$ (none of which is included in the minimal setup).

\footnotetext{
${ }^{9}$ Irreducible breaking terms of higher rank are difficult to realise in explicit models.
} 
Analysing the number of independent operators with all these spurions is quite straightforward using the results presented in section 3. However, it is less obvious how to define a consistent power-counting. Indeed in the minimal setup the size of the spurions can be directly inferred by the structure of the Yukawa couplings (the only exception being $V_{\ell}$, whose size is deduced by imposing $\left.\left|V_{\ell}\right| \sim\left|V_{q}\right|\right)$. Once this is fixed, the minimal setup ensures a CKM-like suppression for all left-handed transitions in the quark sector, as well as the helicity suppression of mixing in the right-handed sector. On the other hand, these two important phenomenological properties are lost if any of the non-minimal spurions listed above can compete, in size, with the two leading breaking terms (without a special alignment in flavour space). It is therefore natural to conclude that if any of the non-minimal spurions are included, they must be quite small in size.

\section{A phenomenological application: LFV at the LHC}

The usefulness of specific hypotheses about symmetry and symmetry-breaking in the flavour sector is quite clear when analysing low-energy flavour-violating observables, both in the quark and in the lepton sector. However, it is worth to stress that the flavour symmetry is quite useful also to simplify and organise analyses of high- $p_{T}$ observables at the LHC. As clearly demonstrated in [29] with the analysis of $p p \rightarrow \mu \bar{\mu}$ data, flavour hypothesis provides a very useful organising principle to sum, with a proper weight, the contributions of different quarks species in $p p$ collisions. To illustrate this statement in the context of the general formalism we have introduced for the $\mathrm{U}(2)^{5}$ SMEFT basis, in the following we briefly discuss how to extract bounds on semi-leptonic four-fermion operators from the Lepton Flavour Violating (LFV) Drell-Yan process $p p \rightarrow \tau \bar{\ell}(\ell=e, \mu)$.

In presence of $d=6$ SMEFT operators, the process $p p \rightarrow \tau \bar{\ell}$ receives tree-level contributions by the partonic scattering $q_{i} \bar{q}_{j} \rightarrow \tau \bar{\ell}$. The quark flavours $\{i, j\}$ appear in the cross-section with a weight that depends on: i) the operators we are considering, ii) the parton distribution functions (PDF) of the colliding protons. Hence, the physical cross-section can be written as the trace over the contraction of two flavour tensors: a "SMEFT tensor", that we denote $F_{q}^{\ell \tau}\left(\left\{C_{i}\right\}\right)$ and that depends on the SMEFT coefficients, and a "PDF tensor", that we denote $K_{q}$. In terms of these two tensor we can write

$$
\sigma(p p \rightarrow \tau \bar{\ell})=\frac{s}{144 \pi \Lambda^{4}} \operatorname{Tr}\left(F_{q}^{\ell \tau}\left(\left\{C_{i}\right\}\right) \cdot K_{q}\right)
$$

where the trace runs over all possible pairs of colliding quarks, as we discuss below, $\Lambda$ is an overall scale that we introduce to normalise the coefficients of the $d=6$ operators, $\sqrt{s}$ is the proton-proton center-of-mass energy, and summation over the index $q=\{u, d\}$ labelling up and down quarks is implied.

- The SMEFT tensor $F_{q}^{\ell \tau}$ is in direct correspondence with the tensor $\Sigma_{\ell q}$ defined in eq. (3.16), controlling the flavour structure of the $Q_{\ell q}^{(1,3)}$ operators, and similar tensors for the other semileptonic operators in table 8. Since our scope is merely illustrative, in the following we will limit ourself to consider only the contributions of $Q_{\ell q}^{(1,3)}$, 
neglecting terms with different helicity. ${ }^{10}$ Separating up and down quark components of $F_{q}^{\ell \tau}$, as well as $\mathrm{SU}(2)_{L}$ singlet and triplet components of $\Sigma_{\ell q}$, we can write

$$
\begin{aligned}
& F_{u}^{\ell \tau n m}=\left|V_{\mathrm{CKM}}^{n r} V_{\mathrm{CKM}}^{m s *}\left(\Sigma_{\ell q}^{(1) \ell \tau, r s}-\Sigma_{\ell q}^{(3) \ell \tau, r s}\right)\right|^{2}, \\
& F_{d}^{\ell \tau n m}=\left|\Sigma_{\ell q}^{(1) \ell \tau, n m}+\Sigma_{\ell q}^{(3) \ell \tau, n m}\right|^{2},
\end{aligned}
$$

where summation over repeated indices is implicit. The $\Sigma_{\ell q}^{(i)}$ appearing in (5.2) are written in the down-quark mass basis, whereas the explicit expression displayed in table 13 is in the interaction basis. After rotating to the down-quark mass-eigenstate basis (see appendix $\mathrm{A}$ ), the entries of $\Sigma_{\ell q}^{(i)}$ relevant to $p p \rightarrow \tau \bar{\mu}$ read:

$$
\begin{aligned}
& \Sigma_{\ell q}^{(i) \mu \tau, d d}=\epsilon_{\ell} e^{i \bar{\phi}_{\ell}} c_{e}\left\{C_{\ell q}^{(i)}\left(\beta_{1}\right)-s_{b} \epsilon_{q} s_{d}^{2}\left[\tilde{C}_{\ell q}^{(i)}\left(\gamma_{1}\right)+\tilde{C}_{\ell q}^{(i)}\left(\gamma_{2}\right)\right]+s_{b}^{2} s_{d}^{2} C_{\ell q}^{(i)}\left(\beta_{2}\right)\right\} \\
& \Sigma_{\ell q}^{(i) \mu \tau, d s}=-\epsilon_{\ell} e^{i \bar{\phi}_{\ell}} c_{e}\left\{s_{b} \epsilon_{q}\left[\tilde{C}_{\ell q}^{(i)}\left(\gamma_{1}\right)+\tilde{C}_{\ell q}^{(i)}\left(\gamma_{2}\right)\right]-s_{b}^{2} C_{\ell q}^{(i)}\left(\beta_{2}\right)\right\} c_{d} s_{d} e^{i \alpha_{d}} \\
& \Sigma_{\ell q}^{(i) \mu \tau, d b}=\epsilon_{\ell} e^{i \bar{\phi}_{\ell}} c_{e}\left\{s_{b}\left[C_{\ell q}^{(i)}\left(\beta_{1}\right)-C_{\ell q}^{(i)}\left(\beta_{2}\right)\right]+\epsilon_{q} \tilde{C}_{\ell q}^{(i)}\left(\gamma_{1}\right)-s_{b}^{2} \epsilon_{q} \tilde{C}_{\ell q}^{(i)}\left(\gamma_{2}\right)\right\} s_{d} e^{i \alpha_{d}} \\
& \Sigma_{\ell q}^{(i) \mu \tau, s d}=-\epsilon_{\ell} e^{i \bar{\phi}_{\ell}} c_{e}\left\{s_{b} \epsilon_{q}\left[\tilde{C}_{\ell q}^{(i)}\left(\gamma_{1}\right)+\tilde{C}_{\ell q}^{(i)}\left(\gamma_{2}\right)\right]-s_{b}^{2} C_{\ell q}^{(i)}\left(\beta_{2}\right)\right\} c_{d} s_{d} e^{-i \alpha_{d}} \\
& \Sigma_{\ell q}^{(i) \mu \tau, s s}=\epsilon_{\ell} e^{i \bar{\phi}_{\ell}} c_{e}\left\{C_{\ell q}^{(i)}\left(\beta_{1}\right)-s_{b} \epsilon_{q} c_{d}^{2}\left[\tilde{C}_{\ell q}^{(i)}\left(\gamma_{1}\right)+\tilde{C}_{\ell q}^{(i)}\left(\gamma_{2}\right)\right]+s_{b}^{2} c_{d}^{2} C_{\ell q}^{(i)}\left(\beta_{2}\right)\right\} \quad(5.3) \\
& \Sigma_{\ell q}^{(i) \mu \tau, s b}=\epsilon_{\ell} e^{i \bar{\phi}_{\ell}} c_{e}\left\{s_{b}\left[C_{\ell q}^{(i)}\left(\beta_{1}\right)-C_{\ell q}^{(i)}\left(\beta_{2}\right)\right]+\epsilon_{q} \tilde{C}_{\ell q}^{(i)}\left(\gamma_{1}\right)-s_{b}^{2} \epsilon_{q} \tilde{C}_{\ell q}^{(i)}\left(\gamma_{2}\right)\right\} c_{d} \\
& \Sigma_{\ell q}^{(i) \mu \tau, b d}=\epsilon_{\ell} e^{i \bar{\phi}_{\ell}} c_{e}\left\{s_{b}\left[C_{\ell q}^{(i)}\left(\beta_{1}\right)-C_{\ell q}^{(i)}\left(\beta_{2}\right)\right]+\epsilon_{q} \tilde{C}_{\ell q}^{(i)}\left(\gamma_{2}\right)-s_{b}^{2} \epsilon_{q} \tilde{C}_{\ell q}^{(i)}\left(\gamma_{1}\right)\right\} s_{d} e^{-i \alpha_{d}} \\
& \Sigma_{\ell q}^{(i) \mu \tau, b s}=\epsilon_{\ell} e^{i \bar{\phi}_{\ell}} c_{e}\left\{s_{b}\left[C_{\ell q}^{(i)}\left(\beta_{1}\right)-C_{\ell q}^{(i)}\left(\beta_{2}\right)\right]+\epsilon_{q} \tilde{C}_{\ell q}^{(i)}\left(\gamma_{2}\right)-s_{b}^{2} \epsilon_{q} \tilde{C}_{\ell q}^{(i)}\left(\gamma_{1}\right)\right\} c_{d} \\
& \Sigma_{\ell q}^{(i) \mu \tau, b b}=\epsilon_{\ell} e^{i \bar{\phi}_{\ell}} c_{e}\left\{C_{\ell q}^{(i)}\left(\beta_{2}\right)+s_{b} \epsilon_{q}\left[\tilde{C}_{\ell q}^{(i)}\left(\gamma_{1}\right)+\tilde{C}_{\ell q}^{(i)}\left(\gamma_{2}\right)\right]+s_{b}^{2} C_{\ell q}^{(i)}\left(\beta_{1}\right)\right\}
\end{aligned}
$$

while $\Sigma_{\ell q}^{(i) e \tau, n m}=\left(s_{e} / c_{e}\right) \times \Sigma_{\ell q}^{(i) \mu \tau, n m}$ for $p p \rightarrow \tau \bar{e}$. Here $\tilde{C}_{\ell q}^{(i)}\left(\gamma_{1,2}\right)=$ $C_{\ell q}^{(i)}\left(\gamma_{2}\right) e^{ \pm i\left(\bar{\phi}_{q}-\phi_{q}\right)}$, and the mixing and phase parameters $c_{e}, c_{b}, c_{d}, \phi_{q}$ and $\alpha_{d}$ are defined in appendix A. To simply the expressions we have set $s_{\tau}=0$ and we have neglected terms proportional to $\tilde{C}_{\ell q}^{(i)}(\xi)$, since they always appear suppressed by $\epsilon_{\ell} \epsilon_{q}^{2}$.

- The PDF tensor $K_{q}$ is given by

$$
K_{q}^{m n}=\int \mathrm{d} \tau \tau \mathcal{L}_{q_{m} \bar{q}_{n}}(\tau)
$$

where $\tau \equiv m_{\ell \tau}^{2} / s, m_{\ell \tau}$ being the invariant mass of the lepton pair, and $\mathcal{L}_{q_{m} \bar{q}_{n}}$ are the parton luminosity functions for $\bar{q}_{n} q_{m}$ colliding partons defined by

$$
\mathcal{L}_{q_{m} \bar{q}_{n}}(\tau)=\int_{\tau}^{1} \frac{\mathrm{d} x}{x}\left[f_{q_{m}}\left(x, \mu_{F}\right) f_{\bar{q}_{n}}\left(\tau / x, \mu_{F}\right)+\left(q_{n} \leftrightarrow \bar{q}_{m}\right)\right]
$$

\footnotetext{
${ }^{10}$ In the limit of massless fermions the helicity is conserved and the contributions of the other semilpetonic operators do not interfere with that of $Q_{\ell q}^{(1,3)}$ in the cross section.
} 
in terms of the single parton PDF $f^{a}$ ( $\mu_{F}$ denotes the factorization scale). Again for illustrative purposes, we compute (5.4) by integrating the high-mass tail of $m_{\ell \tau}$ in the range $m_{\ell \tau} \in[1,5] \mathrm{TeV}$ at $\sqrt{s}=13 \mathrm{TeV}$, setting $\mu_{F}=s \tau$ and using the central values of the PDF4LHC15_nnlo_mc PDF set [30] This leads to

$$
K_{u}=\kappa\left(\begin{array}{ccc}
1 & 0.5 & 0 \\
0.03 & 0.01 & 0 \\
0 & 0 & 0
\end{array}\right), \quad K_{d}=\frac{\kappa}{2}\left(\begin{array}{ccc}
1 & 0.6 & 0.3 \\
0.1 & 0.07 & 0.03 \\
0.04 & 0.02 & 0.01
\end{array}\right)
$$

with $\kappa \approx 4.8 \times 10^{-3}$. The third row and column for $K_{u}$ vanishes because the PDF of the top-quark in still negligible at $\sqrt{s}=13 \mathrm{TeV}$.

We have now all the ingredients to compute the traces in (5.1). In the down sector, the explicit calculation yields

$$
\begin{aligned}
& \operatorname{Tr}\left(F_{d}^{\ell \tau} \cdot K_{d}\right)=\sum_{n, m=\{d, s, b\}}\left|\Sigma_{\ell q}^{\ell \tau, n m}\right|^{2} K^{m n} \\
& \propto\left(1.07+0.068 s_{b}^{2}\right)\left|C_{\ell q}^{(1+3)}\left(\beta_{1}\right)\right|^{2}+\left(0.01+0.068 s_{b}^{2}\right)\left|C_{\ell q}^{(1+3)}\left(\beta_{2}\right)\right|^{2} \\
& +0.13 s_{b}^{2} \operatorname{Re}\left[C_{\ell q}^{(1+3)}\left(\beta_{1}\right) C_{\ell q}^{(1+3)}\left(\beta_{2}\right)^{*}\right]-s_{b} \epsilon_{q} \operatorname{Re}\left[0.15 C_{\ell q}^{(1+3)}\left(\beta_{1}\right) \tilde{C}_{\ell q}^{(1+3)}\left(\gamma_{2}\right)^{*}\right. \\
& \left.+0.2 C_{\ell q}^{(1+3)}\left(\beta_{1}\right) \tilde{C}_{\ell q}^{(1+3)}\left(\gamma_{1}\right)^{*}+0.072 C_{\ell q}^{(1+3)}\left(\beta_{2}\right) \tilde{C}_{\ell q}^{(1+3)}\left(\gamma_{2}\right)^{*}+0.024 C_{\ell q}^{(1+3)}\left(\beta_{2}\right) \tilde{C}_{\ell q}^{(1+3)}\left(\gamma_{1}\right)^{*}\right] \\
& +\epsilon_{q}^{2}\left[0.046\left|\tilde{C}_{\ell q}^{(1+3)}\left(\gamma_{2}\right)\right|^{2}+0.022\left|\tilde{C}_{\ell q}^{(1+3)}\left(\gamma_{1}\right)\right|^{2}\right]+\mathcal{O}\left(\epsilon_{q}^{3}\right) \\
& \stackrel{\gamma_{i}=0}{\longrightarrow} \quad\left(1.07+0.068 s_{b}^{2}\right)\left|C_{\ell q}^{(1+3)}\left(\beta_{1}\right)\right|^{2}+\left(0.01+0.068 s_{b}^{2}\right)\left|C_{\ell q}^{(1+3)}\left(\beta_{2}\right)\right|^{2} \\
& \quad+0.13 s_{b}^{2} \operatorname{Re}\left[C_{\ell q}^{(1+3)}\left(\beta_{1}\right) C_{\ell q}^{(1+3)}\left(\beta_{2}\right)^{*}\right],
\end{aligned}
$$

where we used $s_{d} \approx\left|V_{t d}\right| /\left|V_{t s}\right|$ and $c_{d} \approx 1$ (see appendix A), we have factored out the leptonic part, common to all operators, and we have taken into account that $s_{b}=\mathcal{O}\left(\epsilon_{q}\right)$ when neglecting $\mathcal{O}\left(\epsilon_{q}^{3}\right)$ terms. Here and below $C_{\ell q}^{(1 \pm 3)}(F)=C_{\ell q}^{(1)}(F) \pm C_{\ell q}^{(3)}(F)$. Similarly, from the up-quarks we get

$$
\begin{aligned}
& \operatorname{Tr}\left(F_{u}^{\ell \tau} \cdot K_{u}\right)=\sum_{n, m=\{u, c\}}\left|V_{\mathrm{CKM}}^{n r} V_{\mathrm{CKM}}^{m s *} \Sigma_{\ell q}^{\ell \tau, r s}\right|^{2} K^{m n} \\
& \propto 1.01\left|C_{\ell q}^{(1-3)}\left(\beta_{1}\right)\right|^{2}-\left(0.003 s_{b}-0.035 s_{b}^{2}\right) \operatorname{Re}\left[C_{\ell q}^{(1-3)}\left(\beta_{1}\right) C_{\ell q}^{(1-3)}\left(\beta_{2}\right)^{*}\right] \\
& -0.036 s_{b} \epsilon_{q} \operatorname{Re}\left[C_{\ell q}^{(1-3)}\left(\beta_{1}\right) \tilde{C}_{\ell q}^{(1-3)}\left(\gamma_{2}\right)^{*}+C_{\ell q}^{(1-3)}\left(\beta_{1}\right) \tilde{C}_{\ell q}^{(1-3)}\left(\gamma_{1}\right)^{*}\right]+\mathcal{O}\left(\epsilon_{q}^{3}\right) \text {, }
\end{aligned}
$$

where we have implemented the numerical values of the CKM matrix from [31] and, consistently with the assumption of neglecting $\mathcal{O}\left(\epsilon_{q}^{3}\right)$ terms, we have neglected numerical entries of $\mathcal{O}\left(10^{-3}\right)$. 
From the above expressions we can draw the following conclusions:

- Operators which are both quark- and lepton-flavour violating (parameterised by the coefficients $\gamma_{i}$ ) contribute with very small numerical coefficients (recall that $\left.\left|\epsilon_{q}\right|,\left|s_{b}\right| \lesssim 10^{-1}\right)$. Once we take into account the corresponding bounds from lowenergy processes, such as $B_{s} \rightarrow \tau \ell$, their impact in the cross-section is negligible. This is a nice virtue of the $\mathrm{U}(2)^{5}$ approach that, contrary to the MFV case, allow us to easily separate flavour-conserving and flavour-violating operators.

- Once quark-violating terms are eliminated, we remain with an expression depending only on $C_{\ell q}^{(i)}\left(\beta_{1}\right)$ and $C_{\ell q}^{(i)}\left(\beta_{2}\right)$, which is quite simple. Here the contribution of $C_{\ell q}^{(i)}\left(\beta_{1}\right)$ is largely dominant receiving contributions by valence quarks collisions $(u \bar{u}, d \bar{d} \rightarrow \tau \bar{\ell}) \cdot{ }^{11}$

- The other virtue of the $\mathrm{U}(2)^{5}$ approach is that of separating the contribution of nontrivial dynamics associated to the third generation, which in many UV completions of the SM is the one associated to larger couplings (or lower effective scales). In our specific example these are the terms proportional to $\left|C_{\ell q}^{(i)}\left(\beta_{2}\right)\right|^{2}$. In this case it is interesting to analyse the relative weight of third-generation partons, which have suppressed PDFs, vs. the flavour-violating terms, which receive contributions from the larger light-quark PDFs. The analytical expression of the terms proportional to $\left|C_{\ell q}^{(i)}\left(\beta_{2}\right)\right|^{2}$ in the cross section is

$$
\begin{aligned}
\left.\operatorname{Tr}\left(F_{d}^{\ell \tau} \cdot K_{d}\right)\right|_{\left|C_{\ell q}^{(i)}\left(\beta_{2}\right)\right|^{2}} \propto & K_{d}^{b b}+\left[K_{d}^{b s}+K_{d}^{s b}+\frac{\left|V_{t d}\right|^{2}}{\left|V_{t s}\right|^{2}}\left(K_{d}^{b d}+K_{d}^{d b}\right)\right] s_{b}^{2} \\
& +\left[K_{d}^{s s}+\frac{\left|V_{t d}\right|^{2}}{\left|V_{t s}\right|^{2}}\left(K_{d}^{d s}+K_{d}^{s d}\right)+\frac{\left|V_{t d}\right|^{4}}{\left|V_{t s}\right|^{4}} K_{d}^{d d}\right] s_{b}^{4} .
\end{aligned}
$$

Given $\left|s_{b}\right| \lesssim 10^{-1}$, and given the entries of $K_{d}$ in (5.6), it is easy to realise that for this specific operator the cross-section is completely dominated by the $b \bar{b} \rightarrow \tau \bar{\ell}$ channel, a conclusion that is not obvious a priori and that follows from the underlying flavour symmetry and symmetry-breaking ansatz.

\section{Conclusions}

In this paper we have analysed how the flavour symmetries $\mathrm{U}(3)^{5}$ and $\mathrm{U}(2)^{5}$, with a minimal set of breaking terms, act as organising principle for the dimension-six operators with flavour quantum numbers in the SMEFT. The main results of our analysis are summarised in table 1 and 6 . As can be seen from table 1 , the $\mathrm{U}(3)^{5}$ symmetry, and the MFV hypothesis, allow us to obtain a drastic reduction in the number of independent terms: this is about 25

\footnotetext{
${ }^{11}$ The coefficients $C_{\ell q}^{(i)}\left(\beta_{1,2}\right)$ also appear in low-energy LFV processes such as $\tau \rightarrow \rho \ell$ and $\Upsilon \rightarrow \bar{\tau} \ell$. A detailed analysis of these low-energy processes is beyond the scope of this work. We simply note that, at present, the low-energy bounds on $C_{\ell q}^{(1+3)}\left(\beta_{1}\right)$ are more stringent (by less than one order of magnitude) than those which can be extracted from $\sigma(p p \rightarrow \tau \bar{\ell})$, whereas collider bounds on $C_{\ell q}^{(1+3)}\left(\beta_{2}\right)$ dominate vs. the low-energy ones [32].
} 
times smaller compared to the case of no flavour symmetry (limiting ourself to the leading terms in the MFV expansion). In the $\mathrm{U}(2)^{5}$ case, which encompass a wider spectrum of newphysics models where the third generation plays a special role, the number of independent terms is roughly three times higher compared to the MFV case (see table 6), but it is still about one order of magnitude smaller compared to the case of no flavour symmetry.

We have also provided a general discussion of why these two sets of flavour hypotheses (i.e. flavour symmetry and symmetry-breaking ansatz) are particularly relevant if we are interested in SMEFT implementations where the bounds on the scale of new physics are not largely saturated by the tight constraints from flavour-violating processes. The two flavour hypotheses we have considered in detail are somehow unique in ensuring a "natural" CKM-like suppression for all left-handed transitions in the quark sector, as well as a double (light-mass $\times \mathrm{CKM}$ ) suppression for mixing in the right-handed quark sector. Variations preserving these properties are possible with the implementation of $\mathrm{U}(1)$ symmetries acting on the third family, as in the case of the $\mathrm{U}(2)^{5} \otimes \mathrm{U}(1)_{b} \otimes \mathrm{U}(1)_{\tau}$ group discussed in some detail in section 4 . In principle, more options are possible in the lepton sector, where we have not made any attempt to link flavour violation in the charged-lepton sector to neutrino mixing, which goes beyond the scope of the present analysis.

To highlight the usefulness of this approach in analysing high- $p_{T}$ observables, and the practical implementation of the classification scheme we have introduced for the SMEFT with minimally broken $\mathrm{U}(2)^{5}$, we have discussed the concrete example of $\sigma(p p \rightarrow \tau \bar{\ell})$. As already shown in [29] for $\sigma(p p \rightarrow \mu \bar{\mu})$, also for $\sigma(p p \rightarrow \tau \bar{\ell})$ the implementation of flavour symmetries provides an essential tool to sum the contributions of the different quarks species appearing in $p p$ collisions, which a priori would involve a large number of independent SMEFT coefficients. This is quite relevant given the growing impact of high- $p_{T}$ data in constraining four-fermion operators and, particularly, the flavour-violating ones [32, 33].

So far, most of the implementations of $\mathrm{U}(3)^{5}$ and $\mathrm{U}(2)^{5}$ symmetries in the SMEFT, or better in subsets of the SMEFT operators, were aimed at analysing low-energy data only. However, the key virtue of both these approach is that of generating an EFT where bounds from flavour-violating and flavour-conserving observables are naturally very complementary, as shown for instance in $[11,29,32]$. Most important, with the implementations of these flavour hypotheses the overall effective scale of the SMEFT can be kept relatively low, making the whole construction quite interesting for phenomenology. We thus believe the classification we have presented in this paper can be a useful first step toward a systematic analysis of both flavour-conserving and flavour-violating observables, at both lowand high-energies, in motivated flavoured versions of the SMEFT.

\section{Acknowledgments}

We thank Riccardo Barbieri, Ilaria Brivio, Admir Greljo, Marco Nardecchia, and Sophie Renner, for useful discussion at different stages of this work. This project has received funding from the European Research Council (ERC) under the European Union's Horizon 2020 research and innovation programme under grant agreement 833280 (FLAY), and by 
the Swiss National Science Foundation (SNF) under contract 200021-159720. The work of K.Y. was also supported in part by the JSPS KAKENHI 18J01459.

\section{A Diagonalization of the Yukawa matrices in $\mathrm{U}(2)^{5}$}

Given the flavor structure of $\mathrm{U}(2)^{5}$, it is convenient to parametrise unitary matrices in the following block form:

$$
W=\left(\begin{array}{c|c}
B & \Theta \\
\hline \Xi^{\dagger} & b
\end{array}\right),
$$

where $W$ is a generic $3 \times 3$ unitary matrix that we decompose into a $2 \times 2$ non-unitary block-matrix $B$, 2-dimensional column vectors $\Xi$ and $\Theta$, and a complex number $b$. Using the unitarity constraint $W^{\dagger} W=W W^{\dagger}=\nVdash$ one can solve some of the blocks, in particular:

$$
b=c_{\theta} e^{-i \phi}, \quad \Xi=-\frac{e^{i \phi}}{c_{\theta}} B^{\dagger} \cdot \Theta
$$

where $c_{\theta}=\cos \theta \equiv \sqrt{1-\Theta^{\dagger} \Theta}$ is an angle and $\phi$ is the unitary phase $\operatorname{Det}(W)=e^{-i \phi}$. The block $B$ can be further decomposed into polar form as $B=(\nVdash-A) \cdot U$, where $U$ is a $2 \times 2$ unitary matrix and $(\nVdash-A)$ is a positive semi-definite hermitian matrix, satisfying $(\nVdash-A)^{2}=\nVdash-\Theta \Theta^{\dagger}$. The hermitian matrix $A$ measures the deviation of the block $B$ from being a unitary submatrix. Solving for $A$ yields:

$$
A=\frac{\Theta \Theta^{\dagger}}{1+c_{\theta}}
$$

With this, one finds an exact expression for an arbitrary $3 \times 3$ unitary matrix in block-form:

$$
W=\left(\begin{array}{c|c}
\nVdash-\frac{\Theta \Theta^{\dagger}}{1+c_{\theta}} & e^{i \phi} \Theta \\
\hline-e^{-i \phi} \Theta^{\dagger} & c_{\theta}
\end{array}\right) \cdot\left(\begin{array}{c|c}
U & \nvdash \\
\hline \nvdash & e^{-i \phi}
\end{array}\right) .
$$

On general grounds, the block vector $\Theta$ can be parametrized with two angles $\theta$ and $\varphi$ and two complex phases:

$$
\Theta=\left(\begin{array}{c}
s_{\theta} s_{\varphi} e^{i \xi_{1}} \\
s_{\theta} c_{\varphi} e^{i \xi_{2}}
\end{array}\right)
$$

In the limit where $\varphi \rightarrow 0\left(\varphi \rightarrow \frac{\pi}{2}\right)$ the angle $\theta$ corresponds to a rotation in the 23-plane (13-plane).

We can use this general decomposition to compute the unitary matrices that diagonalise the Yukawa couplings starting from the interaction basis,

$$
L_{f}^{\dagger} Y_{f} R_{f}=\operatorname{diag}\left(y_{f_{1}}, y_{f_{2}}, y_{f_{3}}\right)
$$

for $f=d, u, e$. The unitary matrices $L_{f}$ and $R_{f}$ are parametrized using (A.4) with blocks $\Theta_{f}^{L, R}, U_{f}^{L, R}$ and $\phi_{f}^{L, R}$, respectively. Following ref. [34], the left handed matrices $L_{f}$ are 
to a very good approximation a product of two consecutive rotations in the 12 and 23 planes. The matrix $U_{f}^{L}$ is parametrized with one angle $\theta_{f}$ (rotation in the 12-plane) and one phase $\alpha_{f}$ as in (3.5). The rotation in the 23-plane is achieved for $\varphi_{f}^{L, R} \approx 0$ in (A.5), we therefore write $\Theta_{d}^{L}=\left(0, s_{b}\right)^{T}, \Theta_{u}^{L}=\left(0, s_{t}\right)^{T}, \Theta_{e}^{L}=\left(0, s_{\tau}\right)^{T}$ (where we set $\xi_{2 f}^{L, R}=0$ without loss of generality). The right-handed unitary matrices $R_{f}$, on the other hand, are to a good approximation just a rotation in the 23-plane, hence $U_{f}^{R} \approx \nVdash$. The right-handed and left-handed angles are related through $\Theta_{d}^{R}=\operatorname{diag}\left(\frac{m_{d}}{m_{b}}, \frac{m_{s}}{m_{b}}\right) \Theta_{d}^{L}, \Theta_{u}^{R}=\operatorname{diag}\left(\frac{m_{u}}{m_{t}}, \frac{m_{c}}{m_{t}}\right) \Theta_{u}^{L}$ and $\Theta_{e}^{R}=\operatorname{diag}\left(\frac{m_{e}}{m_{\tau}}, \frac{m_{\mu}}{m_{\tau}}\right) \Theta_{e}^{L}$. For quarks, the phases can be brought to satisfy $\phi_{d}^{L}=\phi_{d}^{R}=$ $\phi_{u}^{R} \equiv \phi_{q}$, while all leptonic phases can be set to zero. With these settings, neglecting tiny subleading terms, we get [34]

$$
\begin{aligned}
L_{d} & \approx\left(\begin{array}{ccc}
c_{d} & -s_{d} e^{i \alpha_{d}} & 0 \\
s_{d} e^{-i \alpha_{d}} & c_{d} & s_{b} \\
-s_{d} s_{b} e^{-i\left(\alpha_{d}+\phi_{q}\right)} & -c_{d} s_{b} e^{-i \phi_{q}} & e^{-i \phi_{q}}
\end{array}\right), \\
R_{d} & \approx\left(\begin{array}{ccc}
1 & 0 & 0 \\
0 & 1 & \frac{m_{s}}{m_{b}} s_{b} \\
0-\frac{m_{s}}{m_{b}} s_{b} e^{-i \phi_{q}} & e^{-i \phi_{q}}
\end{array}\right), \\
R_{u} & \approx\left(\begin{array}{ccc}
1 & 0 & 0 \\
0 & 1 & \frac{m_{c}}{m_{t}} s_{t} \\
0-\frac{m_{c}}{m_{t}} s_{t} e^{-i \phi_{q}} & e^{-i \phi_{q}}
\end{array}\right), \\
L_{e} & \approx\left(\begin{array}{ccc}
c_{e} & -s_{e} & 0 \\
s_{e} & c_{e} & s_{\tau} \\
-s_{e} s_{\tau} & -c_{e} s_{\tau} & 1
\end{array}\right) \\
R_{e} & \approx\left(\begin{array}{ccc}
1 & 0 & 0 \\
0 & 1 & \frac{m_{\mu}}{m_{\tau}} s_{\tau} \\
0-\frac{m_{\mu}}{m_{\tau}} s_{\tau} & 1
\end{array}\right)
\end{aligned}
$$

with $L_{u}$ constrained by $L_{u}=L_{d} V_{\mathrm{CKM}}^{\dagger}$.

- In the quark sector the following two parameters appearing in $L_{u, d}$ and $R_{u, d}$, remains unconstrained: the real parameter $s_{b}$ and the complex phase $\phi_{q}=\phi_{b}-\phi_{t}$, defined by

$$
\frac{s_{b}}{c_{b}} e^{i \phi_{b}}=x_{b} V_{q}, \quad \frac{s_{t}}{c_{t}} e^{i \phi_{t}}=x_{t} V_{q}, \quad \text { with } \quad\left|s_{t}-s_{b} e^{i \phi_{q}}\right|=\left|V_{c b}\right|,
$$

as well ads the phase of $V_{q}$ (which can be chosen to make $x_{t} V_{q}$ real and positive). On 
the other hand, the parameters of $U_{u(d)}$ are completely determined via the relations

$$
\frac{s_{d}}{c_{d}}=\frac{\left|V_{t d}\right|}{\left|V_{t s}\right|}, \quad \frac{s_{u}}{c_{u}}=\frac{\left|V_{u b}\right|}{\left|V_{c b}\right|}, \quad \alpha_{d}=\arg \left(\frac{V_{t d}^{*}}{V_{t s}^{*}}\right), \quad \alpha_{u}=\arg \left(\frac{V_{u b}}{V_{c b}}\right),
$$

which imply

$$
U_{u} U_{d}^{\dagger}=\left(\begin{array}{cc}
\cos \theta_{c} & \sin \theta_{c} \\
-\sin \theta_{c} & \cos \theta_{c}
\end{array}\right)
$$

where $\sin \theta_{c}=\left|V_{u s}\right|$.

- In the lepton sector both $s_{\tau} / c_{\tau}=\left|x_{\tau} V_{\ell}\right|$ and $s_{e}$ remain unconstrained, together with the phase of $V_{\ell}$ (which can be chosen to make $x_{\tau} V_{\ell}$ real and positive).

\section{B On the number of independent fermion contractions}

Since $\mathrm{U}(N) \sim \mathrm{U}(1) \otimes \mathrm{SU}(N)$, to verify that any of the SMEFT operators we consider transforms as a singlet under the flavour group, we have to ensure two things. First, the total phase associated to the U(1) part of the flavour symmetry must be zero. Second, the operator has to be a singlet of the $\mathrm{SU}(N)$ group. To determine all ways to form singlets under the $\mathrm{SU}(N)$ groups we first consider the tensor products of two (anti-)fermion fields in the $N=3$ and $N=2$ case

$$
\begin{aligned}
& \text { For } \mathrm{SU}(3): \quad 3 \otimes \overline{3}=1 \oplus 8, \quad 3 \otimes 3=\overline{3} \oplus 6, \quad \overline{3} \otimes \overline{3}=3 \oplus \overline{6}, \\
& \text { For } \mathrm{SU}(2): \quad 2 \otimes \overline{2}=1 \oplus 3, \quad 2 \otimes 2=1 \oplus 3, \quad \overline{2} \otimes \overline{2}=1 \oplus 3 .
\end{aligned}
$$

For $\mathrm{SU}(3)$ we only get a singlet by contracting a fermion with an anti-fermion, whereas for $\mathrm{SU}(2)$ this is also obtained for two fermions or two anti-fermions However, additional operators that would be allowed due to that are forbidden by the phase factors, e.g $L L$ or $\bar{L}^{c} L$ have non-vanishing total phase. Thus, the only allowed operators bilinear in the fermion fields have each a fermion and an anti-fermion in both cases.

Given the above consideration, for the operators involving four fermion fields we have to pay special attention only to those with four fermion fields of the same type (i.e. which $Q_{\ell \ell}, Q_{q q}^{(1)}, Q_{q q}^{(3)}, Q_{e e}, Q_{u u}$ and $\left.Q_{d d}\right)$. In the following we will consider $Q_{\ell \ell}$ as representative example. In the $N=2$ case, at leading order in the spurions, we have different choices for the contraction of the flavour indices

$$
(i): \quad\left(\bar{L}^{r} L^{r}\right)\left(\bar{L}^{s} L^{s}\right), \quad(i i): \quad\left(\bar{L}^{r} L^{s}\right)\left(\bar{L}^{s} L^{r}\right), \quad(i i i): \quad \epsilon^{r_{1} r_{2}} \epsilon^{s_{1} s_{2}}\left(\bar{L}^{r_{1}} L^{s_{1}}\right)\left(\bar{L}^{r_{2}} L^{s_{2}}\right),
$$

where the letter corresponds to the contraction of both fundamental representations and of both anti-fundamental, and $\epsilon^{a, b}$ is the totally anti-symmetric tensor. However, the decomposition into irreducible representations of the full tensor product for this type of operator yields

$$
(2 \otimes \overline{2}) \otimes(2 \otimes \overline{2})=1 \oplus 1 \oplus 3 \oplus 3 \oplus 3 \oplus 5
$$


and only contains two singlets. We can associate the two singlets to the combinations $(i)$ and (ii) in eq. (B.2) and we deduce that all other choices, such as (iii), must be linear combinations of these two. The same holds also in the $N=3$ case, where

$$
(3 \otimes \overline{3}) \otimes(3 \otimes \overline{3})=1 \oplus 1 \oplus 8 \oplus 8 \oplus 8 \oplus 8 \oplus 10 \oplus \overline{10} \oplus 27 .
$$

and the we two singlets in can be identified with the contractions of the type $(i)$ and $(i i)$ in eq. (B.2).

Operators with the insertion of spurions proceed in a similar manner. In the $\mathrm{SU}(2)$ case a more complicated tensor decomposition occurs at order $\mathcal{O}\left(V^{2}\right)$ for operators with four identical left-handed fields, such as $(\bar{L} L)(\bar{L} L)$. Here we find

$$
(2 \otimes \overline{2})^{3}=1 \oplus 1 \oplus 1 \oplus 1 \oplus 1 \oplus 3 \oplus 3 \oplus 3 \oplus 3 \oplus 3 \oplus 3 \oplus 3 \oplus 3 \oplus 3 \oplus 5 \oplus 5 \oplus 5 \oplus 5 \oplus 5 \oplus 7
$$

hence we need to identify, a priopri, five independent singlet contractions. Taking into account that

$$
\left(V_{\ell} V_{\ell}^{\dagger}\right) \otimes(\bar{L} L) \otimes(\bar{L} L) \sim(1 \oplus 3)_{V} \otimes(1 \oplus 3)_{L_{1}} \otimes(1 \oplus 3)_{L_{2}}
$$

leads to identify the five contractions as follows

$$
\begin{aligned}
(i): & 1_{V} \otimes 1_{L_{1}} \otimes 1_{L_{2}} \sim V_{\ell}^{r} V_{\ell}^{\dagger r}\left(\bar{L}^{s} L^{s}\right)\left(\bar{L}^{t} L^{t}\right), \\
(i i): & 1_{V} \otimes 3_{L_{1}} \otimes 3_{L_{2}} \sim V_{\ell}^{r} V_{\ell}^{\dagger r}\left(\bar{L}^{s} L^{t}\right)\left(\bar{L}^{t} L^{s}\right), \\
(i i i): & 3_{V} \otimes 1_{L_{1}} \otimes 3_{L_{2}} \sim V_{\ell}^{r} V_{\ell}^{\dagger s}\left(\bar{L}^{t} L^{t}\right)\left(\bar{L}^{r} L^{s}\right), \\
(i v): & 3_{V} \otimes 3_{L_{1}} \otimes 1_{L_{2}} \sim V_{\ell}^{r} V_{\ell}^{\dagger s}\left(\bar{L}^{r} L^{s}\right)\left(\bar{L}^{t} L^{t}\right), \\
(v): & 3_{V} \otimes 3_{L_{1}} \otimes 3_{L_{2}} \sim V_{\ell}^{r} V_{\ell}^{\dagger s}\left(\bar{L}^{r} L^{t}\right)\left(\bar{L}^{t} L^{s}\right) .
\end{aligned}
$$

The first two terms are proportional to leading order operators, while (iii) and (iv) are equivalent. Hence, also in this case we have only two independent contractions that we can identify with the terms $(i i i)$ and $(v)$. 


\section{Summary tables}

5-7: Fermion Bilinears

\begin{tabular}{|cc|cc|cc|cc|}
\hline \multicolumn{6}{|c|}{ non-hermitian $(\bar{L} R)$} \\
\hline 5: & $\psi^{2} H^{3}+$ h.c. & \multicolumn{5}{|c|}{$6: \psi^{2} X H+$ h.c. } \\
\hline$Q_{e H}$ & $\left(H^{\dagger} H\right)\left(\bar{\ell}_{p} e_{r} H\right)$ & $Q_{e W}$ & $\left(\bar{\ell}_{p} \sigma^{\mu \nu} e_{r}\right) \tau^{I} H W_{\mu \nu}^{I}$ & $Q_{u G}$ & $\left(\bar{q}_{p} \sigma^{\mu \nu} T^{A} u_{r}\right) \tilde{H} G_{\mu \nu}^{A}$ & $Q_{d G}$ & $\left(\bar{q}_{p} \sigma^{\mu \nu} T^{A} d_{r}\right) H G_{\mu \nu}^{A}$ \\
$Q_{u H}$ & $\left(H^{\dagger} H\right)\left(\bar{q}_{p} u_{r} \tilde{H}\right)$ & $Q_{e B}$ & $\left(\bar{\ell}_{p} \sigma^{\mu \nu} e_{r}\right) H B_{\mu \nu}$ & $Q_{u W}$ & $\left(\bar{q}_{p} \sigma^{\mu \nu} u_{r}\right) \tau^{I} \tilde{H} W_{\mu \nu}^{I}$ & $Q_{d W}$ & $\left(\bar{q}_{p} \sigma^{\mu \nu} d_{r}\right) \tau^{I} H W_{\mu \nu}^{I}$ \\
$Q_{d H}$ & $\left(H^{\dagger} H\right)\left(\bar{q}_{p} d_{r} H\right)$ & & & $Q_{u B}$ & $\left(\bar{q}_{p} \sigma^{\mu \nu} u_{r}\right) \tilde{H} B_{\mu \nu}$ & $Q_{d B}$ & $\left(\bar{q}_{p} \sigma^{\mu \nu} d_{r}\right) H B_{\mu \nu}$ \\
\hline
\end{tabular}

\begin{tabular}{|c|c|c|}
\hline \multicolumn{3}{|c|}{ hermitian $\left(+Q_{H u d}\right) \sim 7: \psi^{2} H^{2} D$} \\
\hline$(\bar{L} L)$ & $(\bar{R} R)$ & $\left(\bar{R} R^{\prime}\right)$ \\
\hline$Q_{H \ell}^{(1)} \quad\left(H^{\dagger} i \overleftrightarrow{D}_{\mu} H\right)\left(\bar{\ell}_{p} \gamma^{\mu} \ell_{r}\right)$ & $Q_{H e} \quad\left(H^{\dagger} i \overleftrightarrow{D}_{\mu} H\right)\left(\bar{e}_{p} \gamma^{\mu} e_{r}\right)$ & $Q_{H u d}+$ h.c. $\quad i\left(\tilde{H}^{\dagger} D_{\mu} H\right)\left(\bar{u}_{p} \gamma^{\mu} d_{r}\right)$ \\
\hline$Q_{H \ell}^{(3)} \quad\left(H^{\dagger} i \overleftrightarrow{D}_{\mu}^{I} H\right)\left(\bar{\ell}_{p} \tau^{I} \gamma^{\mu} \ell_{r}\right)$ & $Q_{H u} \quad\left(H^{\dagger} i \overleftrightarrow{D}_{\mu} H\right)\left(\bar{u}_{p} \gamma^{\mu} u_{r}\right)$ & \\
\hline$Q_{H q}^{(1)} \quad\left(H^{\dagger} i \overleftrightarrow{D}_{\mu} H\right)\left(\bar{q}_{p} \gamma^{\mu} q_{r}\right)$ & $Q_{H d} \quad\left(H^{\dagger} i \overleftrightarrow{D}_{\mu} H\right)\left(\bar{d}_{p} \gamma^{\mu} d_{r}\right)$ & \\
\hline$Q_{H q}^{(3)} \quad\left(H^{\dagger} i \overleftrightarrow{D_{\mu}^{I}} H\right)\left(\bar{q}_{p} \tau^{I} \gamma^{\mu} q_{r}\right)$ & & \\
\hline
\end{tabular}

8: Fermion Quadrilinears

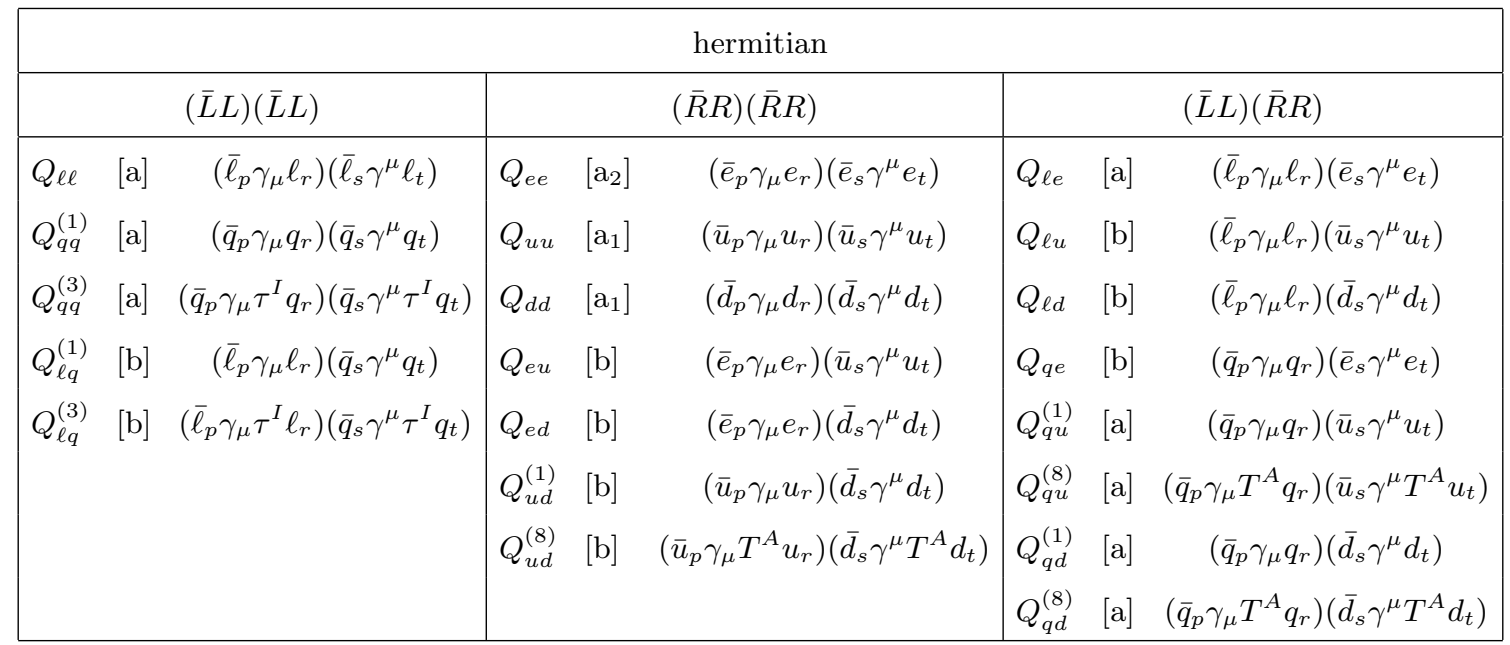

\begin{tabular}{|c|c|c|c|}
\hline \multicolumn{4}{|c|}{ non-hermitian } \\
\hline$(\bar{L} R)(\bar{R} L)+$ h.c. & \multicolumn{3}{|c|}{$(\bar{L} R)(\bar{L} R)+$ h.c. } \\
\hline$Q_{\ell e d q} \quad[\mathrm{a}] \quad\left(\bar{\ell}_{p}^{j} e_{r}\right)\left(\bar{d}_{s} q_{t j}\right)$ & $Q_{q u q c}^{(1)}$ & {$[\mathrm{b}]$} & $\left(\bar{q}_{p}^{j} u_{r}\right) \epsilon_{j k}\left(\bar{q}_{s}^{k} d_{t}\right)$ \\
\hline & $Q_{q u q c}^{(8)}$ & {$[\mathrm{b}]$} & $\left(\bar{q}_{p}^{j} T^{A} u_{r}\right) \epsilon_{j k}\left(\bar{q}_{s}^{k} T^{A} d_{t}\right)$ \\
\hline & $Q_{\ell e q u}^{(1)}$ & {$[a]$} & $\left(\bar{\ell}_{p}^{j} e_{r}\right) \epsilon_{j k}\left(\bar{q}_{s}^{k} u_{t}\right)$ \\
\hline & $Q_{\text {lequ }}^{(3)}$ & {$[\mathrm{a}]$} & $\left(\bar{\ell}_{p}^{j} \sigma_{\mu \nu} e_{r}\right) \epsilon_{j k}\left(\bar{q}_{s}^{k} \sigma^{\mu \nu} u_{t}\right)$ \\
\hline
\end{tabular}

Table 8. List of all fermionic SMEFT operators in the Warsaw basis [2]. The division in classes is adopted from [5]. The letter in square brackets for the four-fermion operators labels the type of the operators as defined in section 3 . 


\begin{tabular}{|c|c|c|c|c|c|c|c|c|c|c|c|}
\hline \multicolumn{12}{|c|}{ MFV breaking terms } \\
\hline \multirow{2}{*}{$\begin{array}{l}\text { Class } \\
X^{3}, H^{6}, H^{4} D^{2}, X^{2} H^{2}\end{array}$} & \multirow{2}{*}{$\begin{array}{c}\text { No. } \\
15\end{array}$} & \multicolumn{2}{|c|}{$Y_{i}^{0}$} & \multicolumn{2}{|c|}{$Y_{i}^{1}$} & \multicolumn{2}{|c|}{$Y_{u}^{2}$} & \multicolumn{2}{|c|}{$Y_{u}^{1} Y_{d}^{1}$} & \multicolumn{2}{|c|}{$Y_{u}^{2} Y_{d}^{1}$} \\
\hline & & 9 & 6 & - & - & - & - & - & - & - & - \\
\hline$\psi^{2} H^{3}$ & 3 & - & - & 3 & 3 & - & - & - & - & 1 & 1 \\
\hline$\psi^{2} X H$ & 8 & - & - & 8 & 8 & - & - & - & - & 3 & 3 \\
\hline$\psi^{2} H^{2} D$ & 8 & 7 & - & - & - & 3 & - & & 1 & - & - \\
\hline total (bilinear) & 19 & 7 & - & 11 & 11 & 3 & - & 1 & 1 & 4 & 4 \\
\hline$(\bar{L} L)(\bar{L} L)$ & 5 & 8 & - & - & - & 6 & - & - & - & - & - \\
\hline$(\bar{R} R)(\bar{R} R)$ & 7 & 9 & - & - & - & 5 & - & - & - & - & - \\
\hline$(\bar{L} L)(\bar{R} R)$ & 8 & 8 & - & - & - & 10 & - & - & - & - & - \\
\hline$(\bar{L} R)(\bar{R} L)$ & 1 & - & - & - & - & - & - & - & - & - & - \\
\hline$(\bar{L} R)(\bar{L} R)$ & 4 & - & - & - & - & - & - & 4 & 4 & - & - \\
\hline total (quadrilinear): & 25 & 25 & - & - & - & 21 & - & 4 & 4 & - & - \\
\hline total: & 59 & 41 & 6 & 11 & 11 & 24 & - & 5 & 5 & & 4 \\
\hline
\end{tabular}

Table 9. Number of independent operators in the SMEFT for the MFV scenario with insertions of different combinations of spurion. 


\begin{tabular}{|c|c|c|c|c|c|c|c|c|c|c|c|c|c|c|}
\hline \multicolumn{15}{|c|}{$\mathrm{U}(2)^{5}$ breaking terms } \\
\hline \multirow{2}{*}{\multicolumn{2}{|c|}{$\begin{array}{lc}\text { Class } & \text { Type } \\
X^{3}, H^{6}, H^{4} D^{2}, X^{2} H^{2}\end{array}$}} & \multirow{2}{*}{$\frac{\text { No. }}{15}$} & \multicolumn{2}{|c|}{$V^{0}$} & \multicolumn{2}{|c|}{$V^{1}$} & \multicolumn{2}{|c|}{$V^{2}$} & \multicolumn{2}{|c|}{$\Delta^{1}$} & \multicolumn{2}{|c|}{$\Delta^{1} V^{1}$} & \multicolumn{2}{|c|}{$V^{3}$} \\
\hline & & & 9 & 6 & - & - & - & - & - & - & - & - & - & - \\
\hline$\psi^{2} H^{3}$ & & 3 & 3 & 3 & 3 & 3 & - & - & 3 & 3 & 3 & 3 & - & - \\
\hline$\psi^{2} X H$ & & 8 & 8 & 8 & 8 & 8 & - & - & 8 & 8 & 8 & 8 & - & - \\
\hline \multirow{4}{*}{$\psi^{2} H^{2} D$} & $(\bar{L} L)$ & 4 & 8 & - & 4 & 4 & 4 & - & - & - & - & - & - & - \\
\hline & $(\bar{R} R)$ & 3 & 6 & - & - & - & - & - & - & - & 3 & 3 & - & - \\
\hline & $Q_{\text {Hud }}$ & 1 & 1 & 1 & - & - & - & - & - & - & 2 & 2 & - & - \\
\hline & total: & 8 & 15 & 1 & 4 & 4 & 4 & - & - & - & 5 & 5 & - & - \\
\hline \multicolumn{2}{|c|}{ total (bilinear): } & 19 & 26 & 12 & 15 & 15 & 4 & - & 11 & 11 & 16 & 16 & - & - \\
\hline \multirow{3}{*}{$(\bar{L} L)(\bar{L} L)$} & $\mathrm{a}:$ & 3 & 15 & - & 9 & 9 & 15 & 3 & - & - & - & - & 3 & 3 \\
\hline & $\mathrm{b}:$ & 2 & 8 & - & 8 & 8 & 12 & 4 & - & - & - & - & 4 & 4 \\
\hline & total: & 5 & 23 & - & 17 & 17 & 27 & 7 & - & - & - & - & 7 & 7 \\
\hline \multirow{4}{*}{$(\bar{R} R)(\bar{R} R)$} & $\mathrm{a}_{1}:$ & 2 & 10 & - & - & - & - & - & - & - & 6 & 6 & - & - \\
\hline & $\mathrm{a}_{2}:$ & 1 & 3 & - & - & - & - & - & - & - & 2 & 2 & - & - \\
\hline & b: & 4 & 16 & - & - & - & - & - & - & 一 & 16 & 16 & - & - \\
\hline & total: & 7 & 29 & - & - & - & - & - & - & - & 24 & 24 & - & - \\
\hline \multirow{3}{*}{$(\bar{L} L)(\bar{R} R)$} & $\mathrm{a}:$ & 5 & 20 & - & 10 & 10 & 10 & - & 5 & 5 & 15 & 15 & - & - \\
\hline & $\mathrm{b}:$ & 3 & 12 & - & 6 & 6 & 6 & - & - & - & 6 & 6 & - & - \\
\hline & total: & 8 & 32 & - & 16 & 16 & 16 & - & 5 & 5 & 21 & 21 & - & - \\
\hline \multicolumn{2}{|l|}{$(\bar{L} R)(\bar{R} L)$} & 1 & 1 & 1 & 2 & 2 & 1 & 1 & 2 & 2 & 4 & 4 & - & - \\
\hline \multirow{3}{*}{$(\bar{L} R)(\bar{L} R)$} & $\mathrm{a}:$ & 2 & 2 & 2 & 4 & 4 & 2 & 2 & 8 & 8 & 12 & 12 & - & - \\
\hline & $\mathrm{b}:$ & 2 & 2 & 2 & 4 & 4 & 2 & 2 & 4 & 4 & 8 & 8 & - & - \\
\hline & total: & 4 & 4 & 4 & 8 & 8 & 4 & 4 & 12 & 12 & 20 & 20 & - & - \\
\hline \multicolumn{2}{|c|}{ total (quadrilinear): } & 25 & 89 & 5 & 43 & 43 & 48 & 12 & 19 & 19 & 69 & 69 & 7 & 7 \\
\hline \multicolumn{2}{|c|}{ total: } & 59 & 124 & 23 & 58 & 58 & 52 & 12 & 30 & 30 & 85 & 85 & 7 & 7 \\
\hline
\end{tabular}

Table 10. Number of independent operators in the SMEFT with $\mathrm{U}(2)^{5}$ flavour symmetry and insertions of different combinations of spurion. 


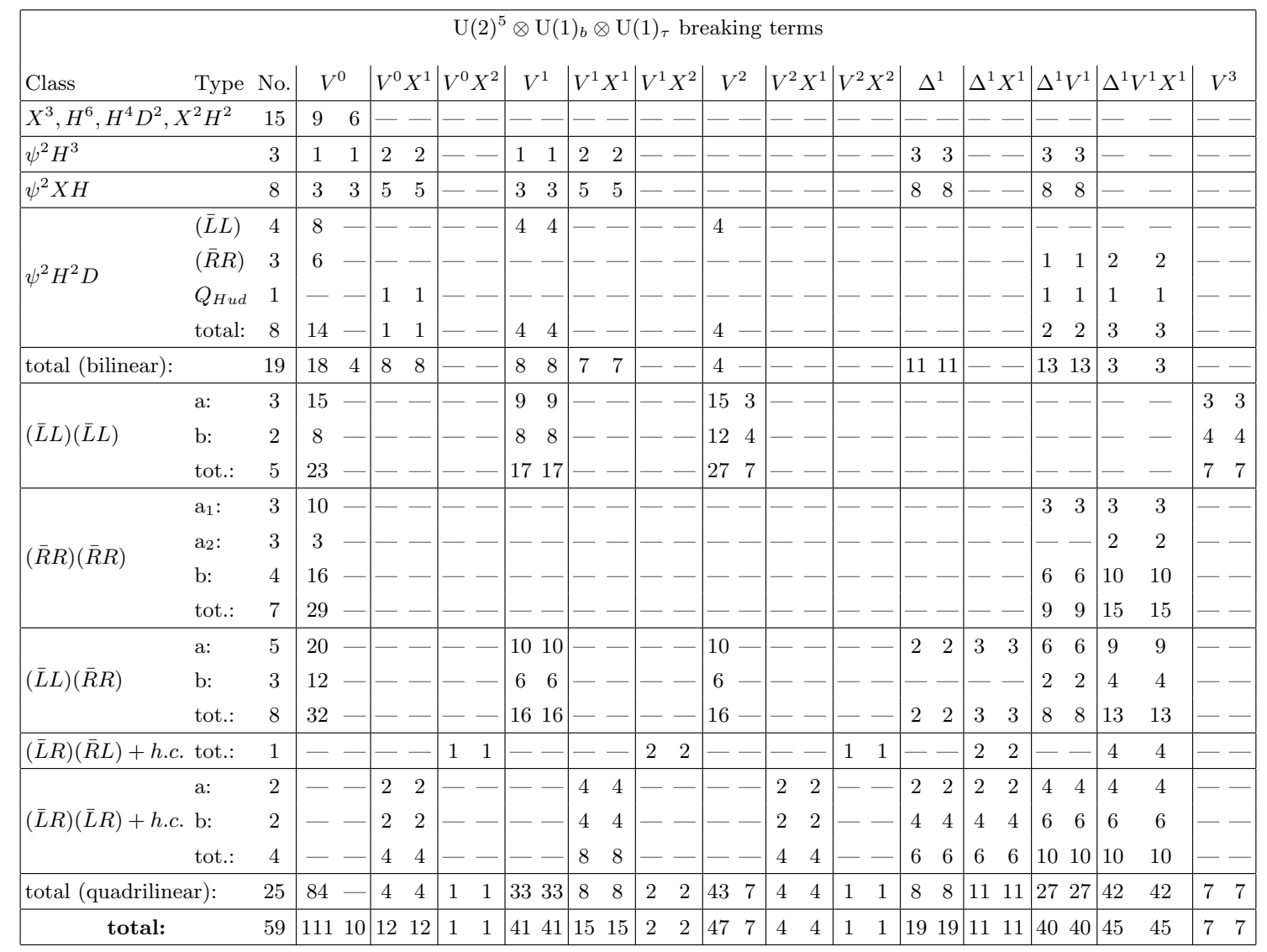

Table 11. Number of independent operators in the SMEFT with $\mathrm{U}(2)^{5} \otimes \mathrm{U}(1)^{2}$ flavour symmetry and insertions of different combinations of spurion. 
(11)

(12)

(13)

(21)

(22)

(23)

(31)

(32)

(33)

(11)

\begin{tabular}{|c|c|c|c|c|c|c|}
\hline $\begin{array}{l}a_{1} \\
a_{2}\end{array}$ & & $\begin{array}{l}2 a_{1} \\
c_{1} \epsilon_{\ell}^{2}\end{array}$ & $\beta_{1} \epsilon_{\ell}$ & & $\beta_{1}^{*} \epsilon_{\ell}$ & $a_{3}$ \\
\hline & $\begin{array}{l}2 a_{2} \\
c_{4} \epsilon_{\ell}^{2}\end{array}$ & & & $\beta_{3}^{*} \epsilon_{\ell}$ & & \\
\hline
\end{tabular}

(13)

(21)

(22)

(23)

\begin{tabular}{|l|l|l|l|l|l|l|l|l|}
\hline & & & $\beta_{3} \epsilon_{\ell}$ & & & $a_{4}$ & & \\
\hline & & & & & & & \\
\hline
\end{tabular}

(31)

(32)

\begin{tabular}{|l|l|l|l|l|l|l|}
$2 a_{2}$ & $\beta_{3} \epsilon_{\ell}$ & & & & & \\
$c_{4} \epsilon_{\ell}^{2}$ & & & & & & \\
\hline
\end{tabular}

(22)

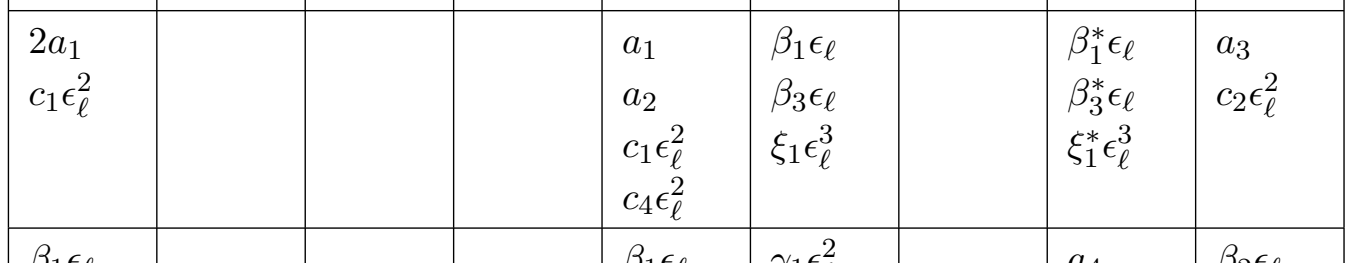

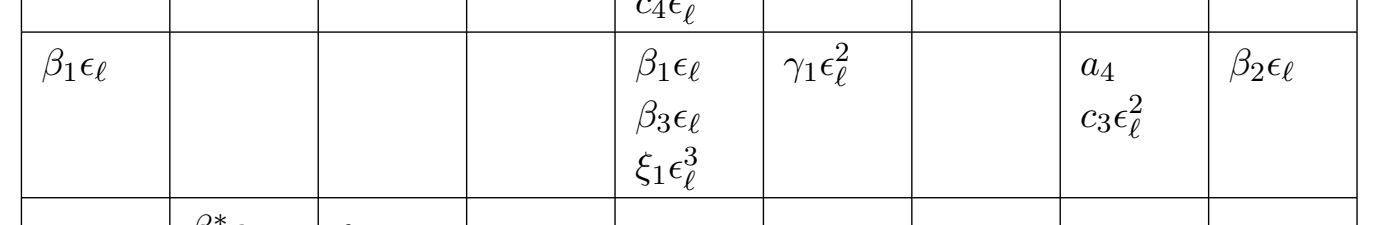

(33)

\begin{tabular}{|c|c|c|c|c|c|c|}
\hline & $\beta_{3}^{*} \epsilon_{\ell}$ & $a_{4}$ & & & & \\
\hline$\beta_{1}^{*} \epsilon_{\ell}$ & & & $\begin{array}{l}\beta_{1}^{*} \epsilon_{\ell} \\
\beta_{3}^{*} \epsilon_{\ell} \\
\xi_{1}^{*} \epsilon_{\ell}^{3}\end{array}$ & $\begin{array}{l}a_{4} \\
c_{3} \epsilon_{\ell}^{2}\end{array}$ & $\gamma_{1}^{*} \epsilon_{\ell}^{2}$ & $\beta_{2}^{*} \epsilon_{\ell}$ \\
\hline$a_{3}$ & & & $\begin{array}{l}a_{3} \\
c_{2} \epsilon_{\ell}^{2}\end{array}$ & $\beta_{2} \epsilon_{\ell}$ & $\beta_{2}^{*} \epsilon_{\ell}$ & $a_{5}$ \\
\hline
\end{tabular}

Table 12. The $\sum_{\ell \ell}^{i j, n m}$ tensor in the interaction basis as defined in eq. (3.16): the entries are as indicated in rows $(\mathrm{ij})$ and columns $(\mathrm{nm})$, respectively. All terms in each cell should be added. 


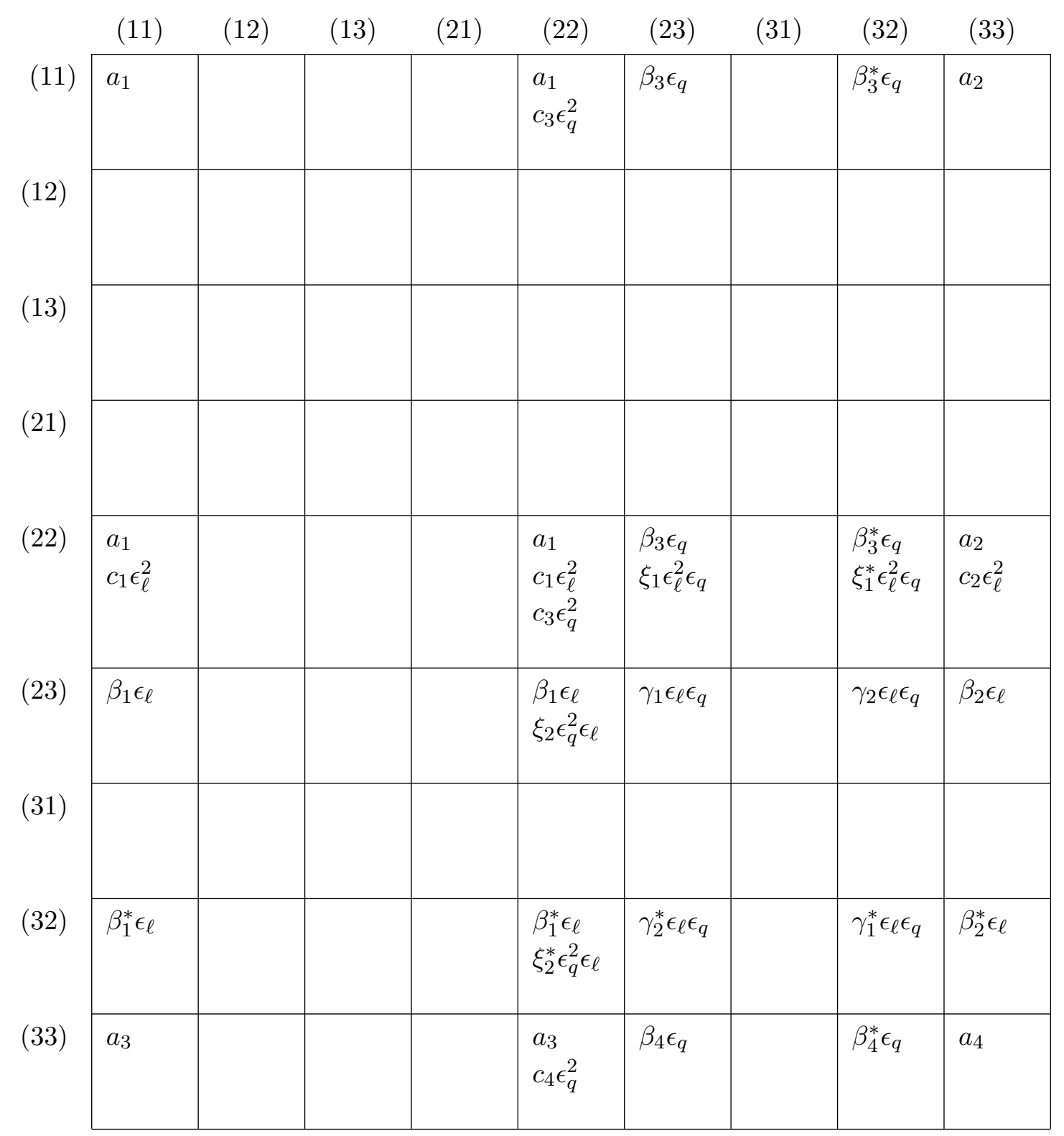

Table 13. The $\Sigma_{\ell q}^{i j, n m}$ tensor in the interaction basis as defined in eq. (3.16): the entries are as indicated in rows $(i j)$ and columns $(\mathrm{nm})$, respectively. All terms in each cell should be added.

Open Access. This article is distributed under the terms of the Creative Commons Attribution License (CC-BY 4.0), which permits any use, distribution and reproduction in any medium, provided the original author(s) and source are credited. 


\section{References}

[1] W. Buchmüller and D. Wyler, Effective Lagrangian analysis of new interactions and flavor conservation, Nucl. Phys. B 268 (1986) 621 [inSPIRE].

[2] B. Grzadkowski, M. Iskrzynski, M. Misiak and J. Rosiek, Dimension-six terms in the Standard Model Lagrangian, JHEP 10 (2010) 085 [arXiv: 1008.4884] [INSPIRE].

[3] E.E. Jenkins, A.V. Manohar and M. Trott, Renormalization group evolution of the Standard Model dimension six operators I: formalism and $\lambda$ dependence, JHEP 10 (2013) 087 [arXiv:1308.2627] [INSPIRE].

[4] E.E. Jenkins, A.V. Manohar and M. Trott, Renormalization group evolution of the Standard Model dimension six operators II: Yukawa dependence, JHEP 01 (2014) 035 [arXiv:1310.4838] [INSPIRE].

[5] R. Alonso, E.E. Jenkins, A.V. Manohar and M. Trott, Renormalization group evolution of the Standard Model dimension six operators III: gauge coupling dependence and phenomenology, JHEP 04 (2014) 159 [arXiv:1312.2014] [INSPIRE].

[6] I. Brivio and M. Trott, The Standard Model as an effective field theory, Phys. Rept. 793 (2019) 1 [arXiv:1706.08945] [INSPIRE].

[7] A. Falkowski, M. González-Alonso and K. Mimouni, Compilation of low-energy constraints on 4-fermion operators in the SMEFT, JHEP 08 (2017) 123 [arXiv:1706.03783] [INSPIRE].

[8] S. Descotes-Genon, A. Falkowski, M. Fedele, M. González-Alonso and J. Virto, The CKM parameters in the SMEFT, JHEP 05 (2019) 172 [arXiv:1812.08163] [INSPIRE].

[9] L. Silvestrini and M. Valli, Model-independent bounds on the Standard Model effective theory from flavour physics, Phys. Lett. B 799 (2019) 135062 [arXiv:1812.10913] [INSPIRE].

[10] A. Falkowski and D. Straub, Flavourful SMEFT likelihood for Higgs and electroweak data, JHEP 04 (2020) 066 [arXiv: 1911.07866] [INSPIRE].

[11] R. Aoude, T. Hurth, S. Renner and W. Shepherd, The impact of flavour data on global fits of the MFV SMEFT, arXiv:2003.05432 [INSPIRE].

[12] A. Helset and A. Kobach, Baryon number, lepton number, and operator dimension in the SMEFT with flavor symmetries, Phys. Lett. B 800 (2020) 135132 [arXiv:1909.05853] [INSPIRE].

[13] G. Isidori, Y. Nir and G. Perez, Flavor physics constraints for physics beyond the Standard Model, Ann. Rev. Nucl. Part. Sci. 60 (2010) 355 [arXiv:1002.0900] [InSPIRE].

[14] R. Chivukula and H. Georgi, Composite technicolor Standard Model, Phys. Lett. B 188 (1987) 99 [INSPIRE].

[15] G. D'Ambrosio, G.F. Giudice, G. Isidori and A. Strumia, Minimal flavor violation: an effective field theory approach, Nucl. Phys. B 645 (2002) 155 [hep-ph/0207036] [InSPIRE].

[16] R. Barbieri, G. Isidori, J. Jones-Perez, P. Lodone and D.M. Straub, U(2) and minimal flavour violation in supersymmetry, Eur. Phys. J. C 71 (2011) 1725 [arXiv:1105.2296] [INSPIRE].

[17] R. Barbieri, D. Buttazzo, F. Sala and D.M. Straub, Flavour physics from an approximate $\mathrm{U}(2)^{3}$ symmetry, JHEP 07 (2012) 181 [arXiv: 1203.4218] [INSPIRE]. 
[18] G. Blankenburg, G. Isidori and J. Jones-Perez, Neutrino masses and LFV from minimal breaking of $\mathrm{U}(3)^{5}$ and $\mathrm{U}(2)^{5}$ flavor symmetries, Eur. Phys. J. C 72 (2012) 2126 [arXiv: 1204.0688] [INSPIRE].

[19] A. Greljo, G. Isidori and D. Marzocca, On the breaking of lepton flavor universality in $B$ decays, JHEP 07 (2015) 142 [arXiv: 1506.01705] [INSPIRE].

[20] R. Barbieri, G. Isidori, A. Pattori and F. Senia, Anomalies in B-decays and U(2) flavour symmetry, Eur. Phys. J. C 76 (2016) 67 [arXiv:1512.01560] [INSPIRE].

[21] D. Buttazzo, A. Greljo, G. Isidori and D. Marzocca, B-physics anomalies: a guide to combined explanations, JHEP 11 (2017) 044 [arXiv: 1706. 07808] [INSPIRE].

[22] C.D. Froggatt and H.B. Nielsen, Hierarchy of quark masses, Cabibbo angles and CP-violation, Nucl. Phys. B 147 (1979) 277 [INSPIRE].

[23] M. Bordone, O. Catà and T. Feldmann, Effective theory approach to new physics with flavour: general framework and a leptoquark example, JHEP 01 (2020) 067 [arXiv: 1910.02641] [INSPIRE].

[24] J.M. Gerard, Fermion mass spectrum in $\mathrm{SU}(2)_{L} \times \mathrm{U}(1)$, Z. Phys. C 18 (1983) 145 [inSPIRE].

[25] I. Brivio, Y. Jiang and M. Trott, The SMEFTsim package, theory and tools, JHEP 12 (2017) 070 [arXiv: 1709.06492] [INSPIRE].

[26] T. Feldmann and T. Mannel, Large top mass and non-linear representation of flavour symmetry, Phys. Rev. Lett. 100 (2008) 171601 [arXiv:0801.1802] [INSPIRE].

[27] A.L. Kagan, G. Perez, T. Volansky and J. Zupan, General minimal flavor violation, Phys. Rev. D 80 (2009) 076002 [arXiv:0903.1794] [InSPIRE].

[28] F. Arias-Aragón, C. Bouthelier-Madre, J.M. Cano and L. Merlo, Data driven flavour model, arXiv: 2003.05941 [INSPIRE].

[29] A. Greljo and D. Marzocca, High-p $p_{T}$ dilepton tails and flavor physics, Eur. Phys. J. C 77 (2017) 548 [arXiv: 1704.09015] [INSPIRE].

[30] J. Butterworth et al., PDF4LHC recommendations for LHC run II, J. Phys. G 43 (2016) 023001 [arXiv: 1510.03865] [INSPIRE].

[31] Particle Data Group collaboration, Review of particle physics, Phys. Rev. D 98 (2018) 030001 [INSPIRE].

[32] A. Angelescu, D.A. Faroughy and O. Sumensari, Lepton flavor violation and dilepton tails at the LHC, Eur. Phys. J. C 80 (2020) 641 [arXiv: 2002.05684] [InSPIRE].

[33] J. Fuentes-Martin, A. Greljo, J. Martin Camalich and J.D. Ruiz-Alvarez, Charm physics confronts high- $p_{T}$ lepton tails, arXiv:2003.12421 [INSPIRE].

[34] J. Fuentes-Martín, G. Isidori, J. Pagès and K. Yamamoto, With or without U(2)? Probing non-standard flavor and helicity structures in semileptonic B decays, Phys. Lett. B 800 (2020) 135080 [arXiv:1909.02519] [INSPIRE]. 